\title{
Importance of using accurate soil profiles for the estimation of pile kinematic input factors*
}

\author{
Guillermo M. Álamoํ, Juan J. Aznárez ${ }^{1}$, Luis A. Padrón ${ }^{1}$, \\ Alejandro E. Martnez-Castro ${ }^{2}$, Orlando Maeso ${ }^{1}$ \\ ${ }^{1}$ Insituto Universitario de Sistemas Inteligentes y Aplicaciones Numéricas en Ingeniería (SIANI), \\ Universidad de Las Palmas de Gran Canaria, Edificio Central del Parque Científico y Tecnológico, \\ Campus Universitario de Tafira 35017 Las Palmas de Gran Canaria, Spain \\ guillermo.alamo / juanjose.aznarez / luis.padron / orlando.maeso @ulpgc.es \\ ${ }^{2}$ Departamento de Mecánica de Estructuras e Ingeniería Hidráulica, ETS de Ingenieros de Caminos, \\ Canales y Puertos, Universidad de Granada, Avenida Fuentenueva s/n, 18002 Granada, Spain \\ amcastro@ugr.es
}

\begin{abstract}
The influence of the variability of the soil profile on the translational and rotational kinematic interaction factors of pile foundations is analysed. Variable-withdepth profiles representative of different soil types are considered, and their results are compared to the ones of equivalent homogeneous soils presenting the same average shear wave velocity. The seismic response of the foundation is computed through an efficient numerical model based on the integral reciprocity theorem in elastodynamics and specific Green's functions for the layered half space. The assumption of the variable soil profile generally leads to a higher filtering effect of the soil lateral displacements, and increases the rotation of the foundation. The importance of the soil variability on the response of the supported structure is also analysed in terms of its pseudo-spectral accelerations. The homogeneous assumption is found to be conservative for structures with low periods, while for other systems, specially the ones with an important contribution of the foundation rotation to the structural response, significantly higher maximum accelerations are obtained when considering the variable profile.
\end{abstract}

Keywords: pile foundations, seismic response, kinematic interaction factors, variable soil profile, integral model

*Pre-print of the paper published in JOURNAL OF GEOTECHNICAL AND GEOENVIRONMENTAL ENGINEERING, 145(8), 04019035 (2019), DOI: 10.1061/(ASCE)GT.1943-5606.0002075 


\section{Introduction}

The study of kinematic interaction factors has been an interesting problem concerning the field of pile foundations for the last decades 1 [5]. These factors represent how the foundation filters the seismic motion of the soil and are generally used as part of substructuring methodologies (see e.g. [6]) in order to study the structural responses.

Several studies have analysed the influence of different variables on the kinematic interaction factors of pile foundations. Mamoon and co-workers [7, 8], followed by Kaynia and Novak [9] and Makris and Badoni [10], highlighted the importance of the wave type and its direction of propagation when computing the foundation seismic motion. More recent works [11, 12] presented kinematic interaction factors for configurations with battered elements, showing the influence of the pile rake angle on the foundation response.

On the other hand, the effects of the variability of the soil profile on the kinematic interaction factors of pile foundations demand more study, specially for the case of pile group configurations. Up to the authors' knowledge, only in the work of Rovithis et al. [13], the interaction factors for a single pile embedded in different soils with depth-varying properties were thoroughly analysed based on a Beam-on-dynamic-Winkler approach. Previous to his work, a brief overview of the higher filtering effects of non-homogeneous soils was also presented by Kaynia and Kausel [14] for the particular cases of a single pile and a $3 \times 3$ pile group in a linearly-variable profile.

This paper wants to help to fill this gap by carrying out a comprehensive study of the influence of the variability of the soil profile on the kinematic interaction factors of several pile foundations. For this purpose, a previously developed numerical model based on the integral reciprocity theorem and the use of specific Green's functions for the layered half space is enhanced. Different variable soil profiles are considered, with its shear wave velocity variation with depth chosen to fit the data extracted from actual boreholes. Then, the influence of considering the actual variable soil profile is analysed by comparing against results obtained by assuming an equivalent homogeneous profile with the same mean properties. Results are presented both in terms of the frequencydependent kinematic interaction factors, and the corresponding elastic response spectra of single degree-of-freedom oscillators attached to the foundation.

\section{Methodology}

The linear dynamic response of the foundation is obtained through a three-dimensional time-harmonic integral numerical model previously developed by the authors [15]. In this model, the soil is assumed to be composed by a finite number of piecewise homogeneous viscoelastic isotropic layers overlying a homogeneous half space and its behaviour is modelled in terms of the reciprocity theorem in elastodynamics and the use of specific Green's functions for this layered half space [16]. The pile-soil interaction tractions are included mathematically as load lines acting within the soil. Piles are modelled as unidimensional Timoshenko's beams through finite elements. Soil and pile formulations are coupled together by imposing compatibility and equilibrium conditions over the pile/soil displacements and the soil-pile interaction tractions, respectively. The use of the advanced Green's functions as fundamental solution and the treatment of piles as load lines (and, 
(a)

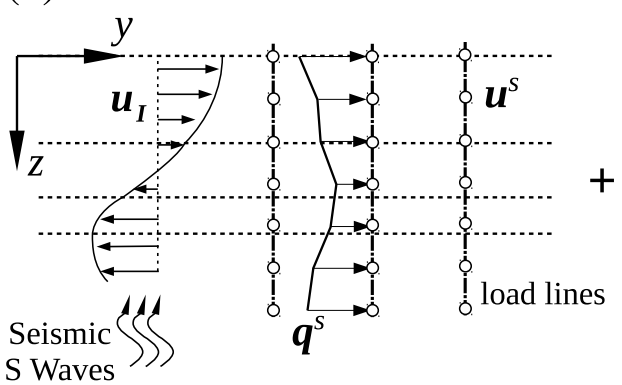

Layered soil

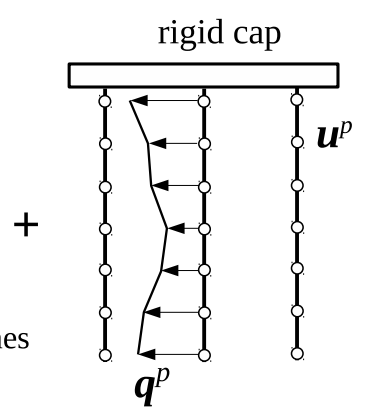

Piles

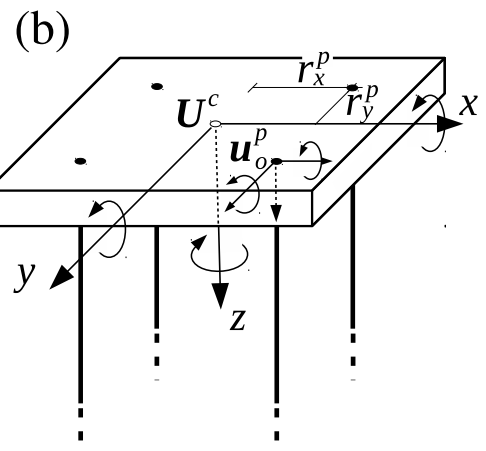

Figure 1: (a) Ilustration of the numerical model used for the analyses. (b) Rigid cap constraints.

therefore, not affecting the soil continuity) results in a very efficient model as it completely avoids the discretization of the free-surface or layer interface variables, yielding a formulation that only depends on the pile variables (displacements and tractions).

This model was used in [15] to study the effects of the soil non-homogeneity on the impedance functions of inclined pile foundations. In the present work, in order to address the seismic problem, the model has been enhanced by including: 1 . the coupling of the piles in a group by a rigid cap, and 2. an incident displacement field as excitation of the system. The model is sketched in Fig 1(a). In the following, a brief overview of the model is presented.

\subsection{Pile Group Equations}

The rigidity of the piles is considered through their beam finite element equations. For this purpose, each pile is discretized into several two-noded elements with 8 unknowns per node corresponding to three displacements, two bending rotations (pile torsional effects are neglected), and three soil-pile interaction tractions. The bending displacements and rotations are modelled inside each element through cubic and quadratic shape functions, respectively, that satisfy the static equation of the Timoshenko's beam; while linear shape functions are used for the axial displacements and for the evolution of the interaction tractions along the element. By following the typical finite element assembly process and assuming harmonic displacements and forces, the pile system of equations results in:

$$
\left(\mathbf{K}\left(1+2 \mathrm{i} \beta_{p}\right)-\omega^{2} \mathbf{M}\right) \mathbf{u}^{\mathbf{p}}-\mathbf{Q q}^{\mathbf{p}}=\mathbf{F}_{\text {top }}
$$

where $\mathbf{K}$ and $\mathbf{M}$ are the global stiffness and mass matrices, $\beta_{p}$ is the hysteretic damping factor of the pile material, $\omega$ is the angular frequency of the excitation, $\mathrm{i}$ is the imaginary unit, $\mathbf{Q}$ is the global matrix that transforms the distributed tractions into the corresponding nodal loads, $\mathbf{u}^{\mathbf{p}}$ and $\mathbf{q}^{\mathbf{p}}$ are the vectors of nodal displacements (and rotations) and soil-pile interaction tractions of the pile, and $\mathbf{F}_{\text {top }}$ is the vector containing the external forces acting at the head of the piles. These external forces can be either prescribed as boundary conditions of the pile head, or produced due to the pile-cap coupling (unknowns of the problem).

For the pile group configurations, each pile in the group is assumed to be fixedly connected to an infinitely-rigid cap. The thickness of the cap is neglected (i.e., the cap 
and all the pile heads are assumed to be located at the same level), as well as the contact between the cap and the soil (i.e., the foundation-soil interaction is produced only along the piles). By considering the rigid body motion of the cap, the displacements and rotations $\left(\mathbf{u}_{o}^{p}=\left\{u_{x}^{p}, u_{y}^{p}, u_{z}^{p}, \theta_{x}^{p}, \theta_{y}^{p}\right\}^{T}\right)$ at the head of each pile $p$ connected to it can be obtained in terms of the three displacements and three rotations of the centre of gravity of the cap $\mathbf{U}^{c}=\left\{U_{x}^{c}, U_{y}^{c}, U_{z}^{c}, \Theta_{x}^{c}, \Theta_{y}^{c}, \Theta_{z}^{c}\right\}^{T}$ as:

$$
\mathbf{u}_{o}^{p}=\mathbf{T}_{c}^{p} \mathbf{U}^{c} \quad \text { being: } \mathbf{T}_{c}^{p}=\left[\begin{array}{cccccc}
1 & 0 & 0 & 0 & 0 & -r_{y}^{p} \\
0 & 1 & 0 & 0 & 0 & r_{x}^{p} \\
0 & 0 & 1 & r_{y}^{p} & -r_{x}^{p} & 0 \\
0 & 0 & 0 & 1 & 0 & 0 \\
0 & 0 & 0 & 0 & 1 & 0
\end{array}\right]
$$

where $r_{j}^{p}$ is the relative distance of the head of pile $p$ with respect to the centre of gravity of the cap in the $j$ direction, as depicted in Fig 1(b).

On the other hand, neglecting the contribution of the inertial effects of the pile cap and assuming that no external forces are acting over it, the equilibrium conditions at the centre of gravity of the pile cap can be directly written in terms of the forces acting on the head of each pile $\mathbf{F}_{\text {top }}^{p}=\left\{f_{x}^{p}, f_{y}^{p}, f_{z}^{p}, m_{x}^{p}, m_{y}^{p}\right\}^{T}$ as:

$$
\sum_{p=1}^{n_{p}}\left(\mathbf{T}_{c}^{p}\right)^{\mathrm{T}} \mathbf{F}_{\text {top }}^{p}=\mathbf{0}
$$

where $n_{p}$ is the number of piles connected to the cap.

\subsection{Soil Equations}

The soil formulation is based on the integral expression of the reciprocity theorem in elastodynamics which, once the boundary conditions of the problem under study and the used fundamental solution are considered, results in:

$$
\mathbf{u}^{\kappa}=-\sum_{l=1}^{n_{p}} \int_{\Gamma_{l}} \mathbf{q}_{l}^{\mathbf{s}} \mathbf{u}^{*} \mathrm{~d} \Gamma_{l}
$$

where $\mathbf{u}^{\kappa}$ is the vector containing the three displacements at the collocation point $\kappa, \Gamma_{l}$ denotes the load line corresponding to pile $l, \mathbf{u}^{*}$ is the tensor containing the displacement Green's functions for the layered half space, and $\mathbf{q}_{l}^{\mathbf{s}}$ are the soil-pile interaction tractions acting over the soil.

Considering the finite element discretization of the piles, and applying Eq. (4) to all pile nodes, the following system of equations is obtained:

$$
\Upsilon \mathbf{u}^{\mathrm{s}}+\mathrm{Gq}^{\mathrm{s}}=\mathbf{0}
$$

where $\mathbf{u}^{\mathbf{s}}$ and $\mathbf{q}^{\mathbf{s}}$ are the vectors containing the displacements and soil interaction tractions at all the pile nodes, $\Upsilon \Upsilon$ is the collocation matrix which contains either identity submatrices or the corresponding pile displacements shape functions depending on whether or not a non-nodal collocation strategy is needed, and $\mathbf{G}$ is the influence matrix obtained by integrating the fundamental solution displacements times the linear shape functions of the interaction tractions along each pile element (see [15] for more details). 


\subsection{Seismic Excitation}

In order to include the seismic excitation, the total displacement field is assumed to be obtained as the superposition of the incident displacement field produced by the seismic waves and the scattered field produced by the presence of the piles [17, 18]. By taking into account that Eq. (4) is expressed in terms of this scattered field, it can be rewritten in order to include the total displacement field as:

$$
\mathbf{u}^{\kappa}=-\sum_{l=1}^{n_{p}} \int_{\Gamma_{l}} \mathbf{q}_{l}^{\mathbf{s}} \mathbf{u}^{*} \mathrm{~d} \Gamma_{l}+\mathbf{u}_{\mathbf{I}}{ }^{\kappa}
$$

Following the same discretization process as before, the contribution of the incident field in Eq. (5) results in:

$$
\Upsilon \mathbf{u}^{\mathrm{s}}+\mathrm{Gq}^{\mathrm{s}}=\mathbf{u}_{\mathbf{I}}
$$

where $\mathbf{u}_{\mathbf{I}}$ is the vector containing the displacements produced by the incident field in all the collocation points.

In the work at hand, the seismic excitation is assumed to be a planar wavefront of shear $(\mathrm{S})$ waves propagating vertically through the layered soil. These waves are assumed to be acting in the $y$ direction, producing a displacement field that is defined for each layer $j$ of the soil as:

$$
\left\{\begin{array}{l}
u_{I_{y}}^{j}(z)=A_{j} \mathrm{e}^{\mathrm{i} k_{j} z}+B_{j} \mathrm{e}^{-\mathrm{i} k_{j} z} \\
u_{I_{x}}^{j}(z)=0 ; \quad u_{I_{z}}^{j}(z)=0
\end{array}\right.
$$

where $A_{j}, B_{j}$ are the frequency-dependent complex amplitudes of the upwards and downwards travelling waves corresponding to layer $j$ that are obtained by solving the unidimensional wave propagation problem, $k_{j}=\omega / c_{s}^{j}$ is the wave number of layer $j, c_{s}^{j}$ is the shear wave velocity of layer $j$, and $z$ is the coordinate that indicates the depth with respect to the free-surface. For normalization purposes, it is useful to define the free-field displacement $u_{f f}$ as the displacement at the free-surface level without considering the presence of the foundation, i.e. $u_{f f}=u_{I_{y}}^{1}(0)$.

\subsection{Coupling Equations}

The pile and soil variables are coupled together by applying compatibility conditions in terms of the displacements at the pile nodes $\left(\mathbf{u}^{\mathbf{s}}=\mathbf{u}^{\mathbf{p}}\right)$ and equilibrium conditions in terms of the soil-pile interaction tractions $\left(\mathbf{q}^{\mathbf{s}}=-\mathbf{q}^{\mathbf{p}}\right)$. By doing so, and combining Eqs. (1), (2), (3) and (7), the following system of linear equations is obtained:

$$
\mathcal{A}\left\{\mathbf{u}^{\mathbf{p}}, \mathbf{q}^{\mathbf{p}}, \mathbf{F}_{\text {top }}, \mathbf{U}^{c}\right\}^{T}=\mathcal{B}\left(\mathbf{u}_{\mathbf{I}}\right)
$$

where $\mathcal{A}$ is the square matrix of coefficients, and $\mathcal{B}$ is the known vector obtained from the rearrangement of the boundary conditions and from the computation of the displacements of the incident field.

\subsection{Verification Results}

In order to verify the capability of the proposed formulation to compute the seismic response of pile foundations, the results presented by Kaynia and Kausel [14] are reproduced. 

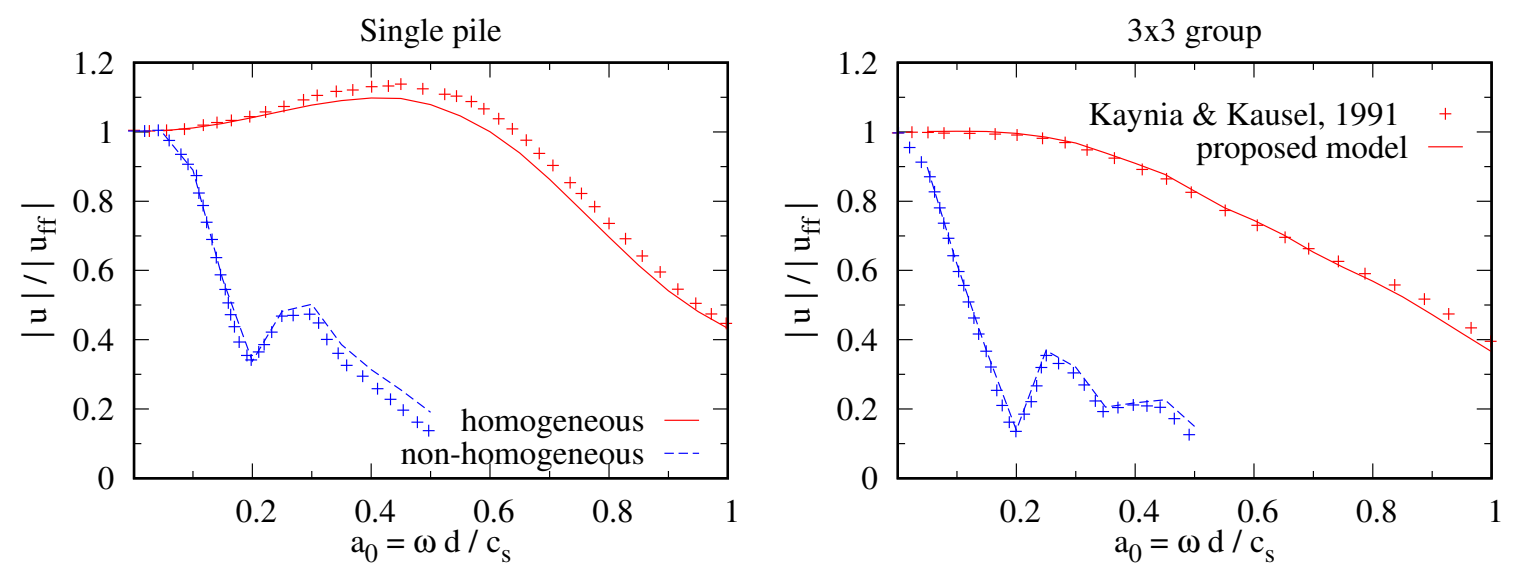

Figure 2: Kinematic interaction factors for different soil profiles. Comparison with the results of Kaynia and Kausel.

In their work, they obtained the ratio between the absolute value of the displacement at the center of the foundation $(u)$ and the free-field displacement for a single pile and a $3 \times 3$ pile group embedded both in a homogeneous half space (pile-soil Young's modulus ratio $\left.E_{p} / E_{s}=100\right)$ and a non-homogeneous soil whose elastic modulus increases linearly with depth going from $E_{p} / E_{s}=0$ at the free-surface level to $E_{p} / E_{s}=100$ at the pile tip and remains constant along the underlying half space. The rest of the properties needed to define the problem are: soil-pile density ratio $\rho_{s} / \rho_{p}=0.7$, soil Poisson's ratio $\nu_{s}=0.4$ and hysteretic damping coefficient $\beta_{s}=5 \%$, pile Poisson's ratio $\nu_{p}=0.25$ and hysteretic damping coefficient $\beta_{p}=0 \%$, pile aspect ratio $L / d=20$ and pile separation distance $s / d=5$ for the group configuration.

Fig. 2 shows the comparison between the displacement kinematic interaction factors obtained by the proposed model and the previous results from the literature. Results for both soil profiles are plotted against the dimensionless frequency $a_{o}=\omega d / c_{s}$ (for the variable profile, the value of the shear wave velocity at the pile tip is assumed). A good agreement is found between the two methodologies both for the single pile and the group configuration. Note that, in order to correctly represent the results of the continuouslyvarying profile, a high-enough number of soil layers is required, which can be determined based on a convergence analysis.

\section{Problem statement}

Pile foundations corresponding to configurations of a single vertical pile and $2 \times 2$ and $3 \times 3$ vertical pile groups are studied in order to fulfil the aims of the present paper. Pile groups composed by a higher number of pile elements were also considered, but their results are not presented for brevity's sake. However, it can be said that the selected results are enough to extrapolate the group behaviour for larger pile configurations.

Different pile geometries are analysed by combining the following parameters representative of typical pile foundations: pile length $L=10,30$ and $50 \mathrm{~m}$, pile diameter $d=0.5$ and $1.5 \mathrm{~m}$, and pile separation distance $s / d=2$ and 5 . In the group configurations, all 

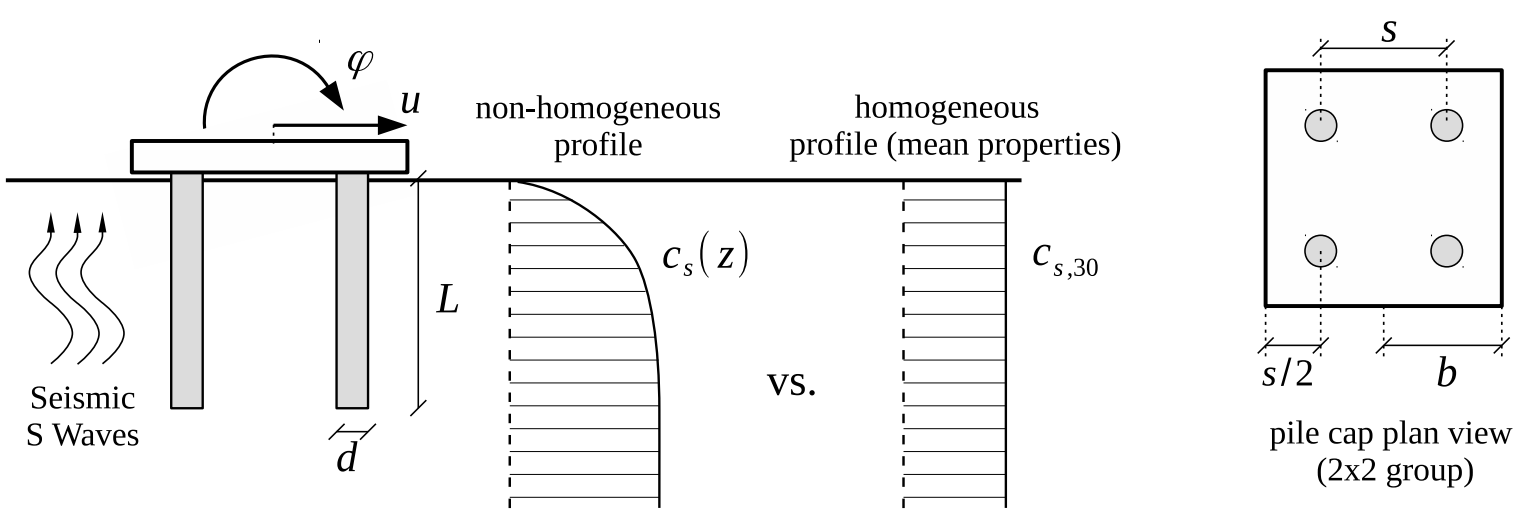

Figure 3: Sketch of the problem under study.

piles are assumed to have the same dimensions. The foundation halfwidth $b$ is considered to be equal to $d, s$ or $3 s / 2$ for the single, $2 \times 2$ and $3 \times 3$ configurations, respectively. A sketch of the problem under study and the representation of these parameters is presented in Fig. 3 .

On the other hand, the material properties of the piles are determined by: Young's modulus $E_{p}=30 \mathrm{GPa}$, density $\rho_{p}=2500 \mathrm{~kg} / \mathrm{m}^{3}$ and Poisson's ratio $\nu_{p}=0.2$. No material damping $\left(\beta_{p}=0 \%\right)$ is considered for the piles. These material properties are chosen in order to represent both solid cross-section concrete piles and hollow steel piles (through equivalent solid cross-section properties). The shear correction factor (Timoshenko's beam theory) $\alpha=0.9$ corresponding to solid cross-sections is considered for the analyses. However, almost no differences are appreciated in the obtained results with respect to the use of the hollow section value $(\alpha=0.5)$.

The soil profiles that are studied in this paper are selected based on the regressed expressions presented by Wang and Wang [19]. They analysed two databases with information about boreholes from California and Japan and obtained the linear and power-law expressions for the shear wave velocity that better adjusted the data depending on the soil type. In the current work, the power-law profiles obtained from the California database are assumed, as these soils are representative of other seismically active areas (e.g. the Mediterranean area). On the other hand, the power-law expressions are used because they presented a better fitting to the empirical data than the linear ones for most cases. Nevertheless, a brief discussion about the use of the linear expressions is presented in the last part of the Results section.

Table 1 presents the different soil types according to the ASCE classification [20, 21], along with the expressions for the varying shear wave velocity obtained by Wang and Wang [19]. The results of these varying profiles are compared with the ones obtained from an equivalent homogeneous half space that has the same average shear wave velocity $c_{s, 30}[20,22]$ as the non-homogeneous profile. This average shear wave velocity is defined in such a way that the shear wave needs the same time to travel along the first $30 \mathrm{~m}$ of the varying profile and of the homogeneous soil. The rest of soil properties are assumed to be constant and equal for both the homogeneous and non-homogeneous profiles. These properties are: soil density $\rho_{s}=1750 \mathrm{~kg} / \mathrm{m}^{3}\left(\rho_{s} / \rho_{p}=0.7\right)$, soil Poisson's ratio $\nu_{s}=0.4$, and soil hysteretic damping coefficient $\beta_{s}=5 \%$. 
Table 1: Soil profiles under study.

\begin{tabular}{ccccccc}
\hline \hline \multicolumn{2}{c}{ Site Class clasification $[20]$} & & \multicolumn{3}{c}{ Regressed expressions from empirical profiles [19] } \\
\cline { 1 - 2 } \cline { 1 - 3 } Type & $c_{s, 30}^{\min }$ & $c_{s, 30}^{\max }$ & & $c_{s}(z)$ & $c_{s, 30}$ & $E_{p} / E_{s}\left(c_{s, 30}\right)$ \\
\hline $\mathrm{C}$ & 360 & 760 & & $242 z^{0.271}$ & 443.4 & $\approx 30$ \\
$\mathrm{D}$ & 180 & 360 & & $126 z^{0.317}$ & 253.0 & $\approx 95$ \\
$\mathrm{E}$ & - & 180 & & $80.9 z^{0.297}$ & 156.2 & $\approx 256$ \\
\hline \hline
\end{tabular}

Note: $\overline{\text { Shear wave velocities in } \mathrm{m} / \mathrm{s} \text {. }}$

In order to simulate the behaviour of the continuously-varying profiles with the proposed methodology, they are discretized into piecewise homogeneous layers with a height of $h_{l}=0.125 \mathrm{~m}$ along the first $50 \mathrm{~m}$ of the soil profile. Below this depth, constant soil properties are assumed for the underlying half space. These values are obtained from a convergence study, and neither increasing the discretization nor the maximum depth alters the presented results.

\section{Results}

\subsection{Kinematic Interaction Factors}

For all pile configurations, the translational and rotational kinematic interaction factors are defined as $I_{u}=u / u_{f f}$ and $I_{\varphi}=\varphi b / u_{f f}$, respectively, being $u$ and $\varphi$ the lateral displacement and rotation at the center of the foundation (see Fig. 3). These kinematic interaction factors are complex-valued and frequency-dependent terms that indicate how the soil seismic motion is transmitted to the supported structure by the foundation. A frequency range between 0 and $160 \mathrm{rad} / \mathrm{s}$ is considered, as the energy content of typical earthquakes lies within this range. Furthermore, this range coincide with the frequencies that a sampling rate of $50 \mathrm{~Hz}$ can capture.

The main scope of the work at hand is to study how the seismic response of different pile foundations changes due to the variability of the soil profile and, therefore, the importance of considering the actual soil profile in the estimation of the group response. For this purpose, the results of the non-homogeneous profile (black lines) are compared with the ones of their equivalent homogeneous half space in terms of $c_{s, 30}$ (blue lines). Results for the stiffest $\left(c_{s, 30}^{\max }\right.$, red lines) and softest $\left(c_{s, 30}^{\min }\right.$, green lines) homogeneous profiles corresponding to each soil type are also included as references.

At this point, it is important to remark that the responses of the three pile geometries with the smallest diameter $(d=0.5 \mathrm{~m})$ are nearly identical. In the same way, the two configurations of diameter $d=1.5 \mathrm{~m}$ and slender piles $(L / d \geq 20)$ also exhibit virtually the same behaviour. For that reason, and in order to present the obtained results in the most compact way, only the kinematic interaction factors corresponding to the representative configurations are displayed. Thus, the results of the configuration $L=30 \mathrm{~m}, d=0.5 \mathrm{~m}$ (labelled as $L 30 d 0.5$ ) also represent the ones of configurations $L=10 \mathrm{~m}, d=0.5 \mathrm{~m}$ and $L=50 \mathrm{~m}, d=0.5 \mathrm{~m}$; while the results corresponding to the configuration $L=30 \mathrm{~m}$, $d=1.5 \mathrm{~m}$ (labelled as $L 30 d 1.5$ ) are also valid for configuration $L=50 \mathrm{~m}, d=1.5 \mathrm{~m}$. 

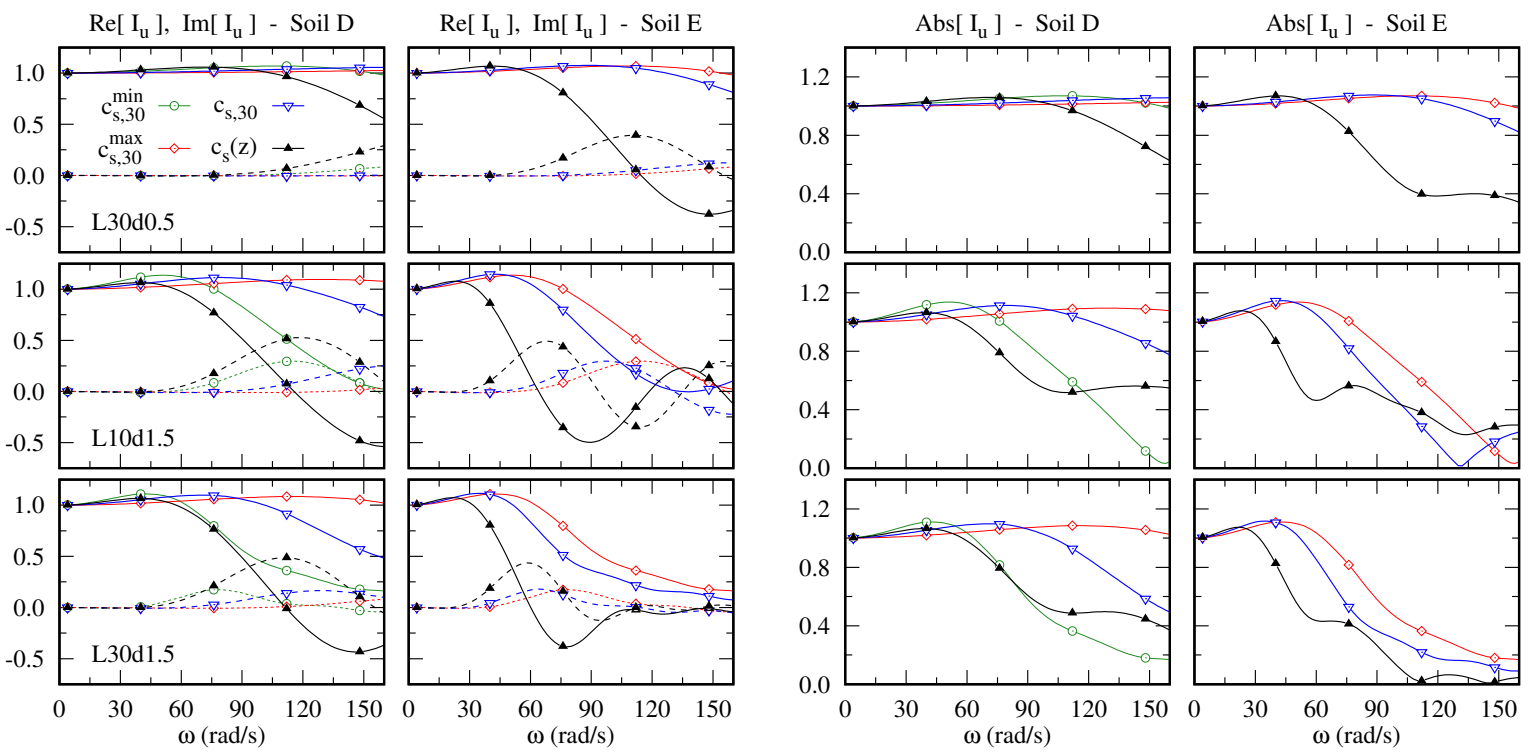

Figure 4: Translational kinematic interaction factors for single piles. (Dashed lines represent imaginary components)

\subsubsection{Translational kinematic interaction factors $\left(I_{u}\right)$}

Fig. 4 presents the translational kinematic interaction factors for the studied single pile foundations embedded in soils of type D and E. The results of the representative configurations are displayed along the three rows. The first column shows the real (solid lines) and imaginary (dashed lines) components of the kinematic interaction factors for soil type D, while their absolute value is presented in the third column. On the other hand, the second and fourth columns present the real/imaginary and absolute values of the kinematic interaction factor for soil type E, respectively.

Attending to the results of soil $\mathrm{D}$, it is found that the real component of $I_{u}$ decays with increasing frequency to a greater extent for the variable profile rather than for its equivalent homogeneous soil (or even for the homogeneous soil with minimum shear velocity). The effect of the variability of the soil profile is also seen for the imaginary component with a higher increment of its value as the frequency augments. Comparing the absolute value of the interaction factors for the non-homogeneous and its equivalent-homogeneous profiles for this soil type, it is found that in the low-frequency range both profiles present nearly the same values (the ones of the varying profile are slightly higher), while for larger frequencies the varying profile filters the ground motion to a greater extent with respect to its homogeneous equivalent profile. These results agree with the findings of previous works [14, 13]. Attending to the classical representation of the interaction factors against the dimensionless frequency, it can be understood that, owing to its higher diameter (and, consequently, higher $a_{o}$ for the same range of $\omega$ ), the configurations with $d=1.5 \mathrm{~m}$ present lower values of $\left|I_{u}\right|$ at smaller frequencies than the foundations with $d=0.5 \mathrm{~m}$.

On the other hand, for the soil type E, the effects of the variability of the profile commented above are intensified. The absolute value of the kinematic interaction factor for the configuration $L 30 d 1.5$ even vanishes for frequencies higher than $110 \mathrm{rad} / \mathrm{s}$ only for the non-homogeneous profile. A singular situation is found in this soil type for the 


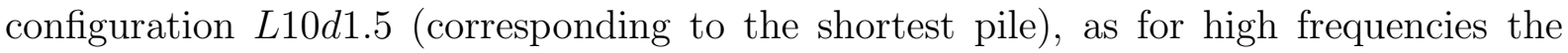
variable profile presents higher $\left|I_{u}\right|$ than its equivalent homogeneous one.

Fig. 5 presents the translational kinematic interaction factors for the $2 \times 2$ pile groups under study. The disposition of the results is the same as the one presented in Fig. 4, with the exception that now two rows per configuration are displayed in order to show the results for the two studied separation distances $s / d$. In general terms, it is found that for pile groups the effects of the variability of the soil profile can be observed at frequencies lower than those for the single pile, since for group configurations the absolute value of the translational kinematic interaction factor is always below unity due to the pile head rotation restriction imposed by the cap. This effect also produces that, for pile groups, the results corresponding to the non-homogeneous profiles are always smaller than the ones of their equivalent homogeneous soil in the low-frequency range.

The absolute values corresponding to the smallest distance between piles $(s / d=2)$ show that, for the L30d0.5 and L10d1.5 configurations, the varying profiles filter the seismic excitation to a greater extent than their $c_{s, 30}$ homogeneous profiles along all the studied frequency range. On the other hand, for configuration L30d1.5 there is an interval of frequencies (100-160 rad/s for soil D, and 45-90 rad/s for type E) in which the nonhomogeneous profiles present higher values of $\left|I_{u}\right|$ than their homogeneous equivalent ones. This effect is mainly produced due to the larger values of the imaginary component of the results for the variable profile, in addition to the fact that their real component reaches more negative values at smaller frequencies for this profile. Also, for this specific configuration ( $L 30 d 1.5$ and $s / d=2$ ), it is important to highlight the large oscillations with the frequency that can be found in the real and imaginary components of the $I_{u}$ in the high-frequency range. These oscillations occur both for the variable and the homogeneous profiles.

Regarding the influence of increasing the separation distance between the piles in the group, it has a minor effect on the L30d0.5 configuration, just slightly increasing the higher filtering effect of the varying profile in the medium-high-frequency range. On the contrary, for the configurations represented by L30d1.5, increasing the distance between the piles significantly diminishes the above-mentioned high oscillations of the real and imaginary components of the $I_{u}$ at large frequencies. This phenomenon may indicate that this oscillatory behaviour is produced by the pile-to-pile interaction between the elements of the group, which is reduced as the distance between piles increases.

Fig. [6 shows now the results for the $3 \times 3$ configurations. The translational kinematic interaction factors and the effects of the soil profile variability and pile separation obtained for these pile groups are analogous to the ones corresponding to the $2 \times 2$ groups. Attending to the results of $L 30 \mathrm{~d} 1.5$, increasing the number of piles in the group is found to augment the oscillations of the real and imaginary components at high frequencies for all soil profiles due to the higher number of piles that can interact with each other.

\subsubsection{Rotational kinematic interaction factors $\left(I_{\varphi}\right)$}

Fig. 7 shows the rotational kinematic interaction factors for the monopile configurations embedded in soil types D and E following the same distribution that was used for the translational factors in the previous section. In general terms, two zones with different behaviours can be distinguished in the obtained results. In the low-frequency range, the 

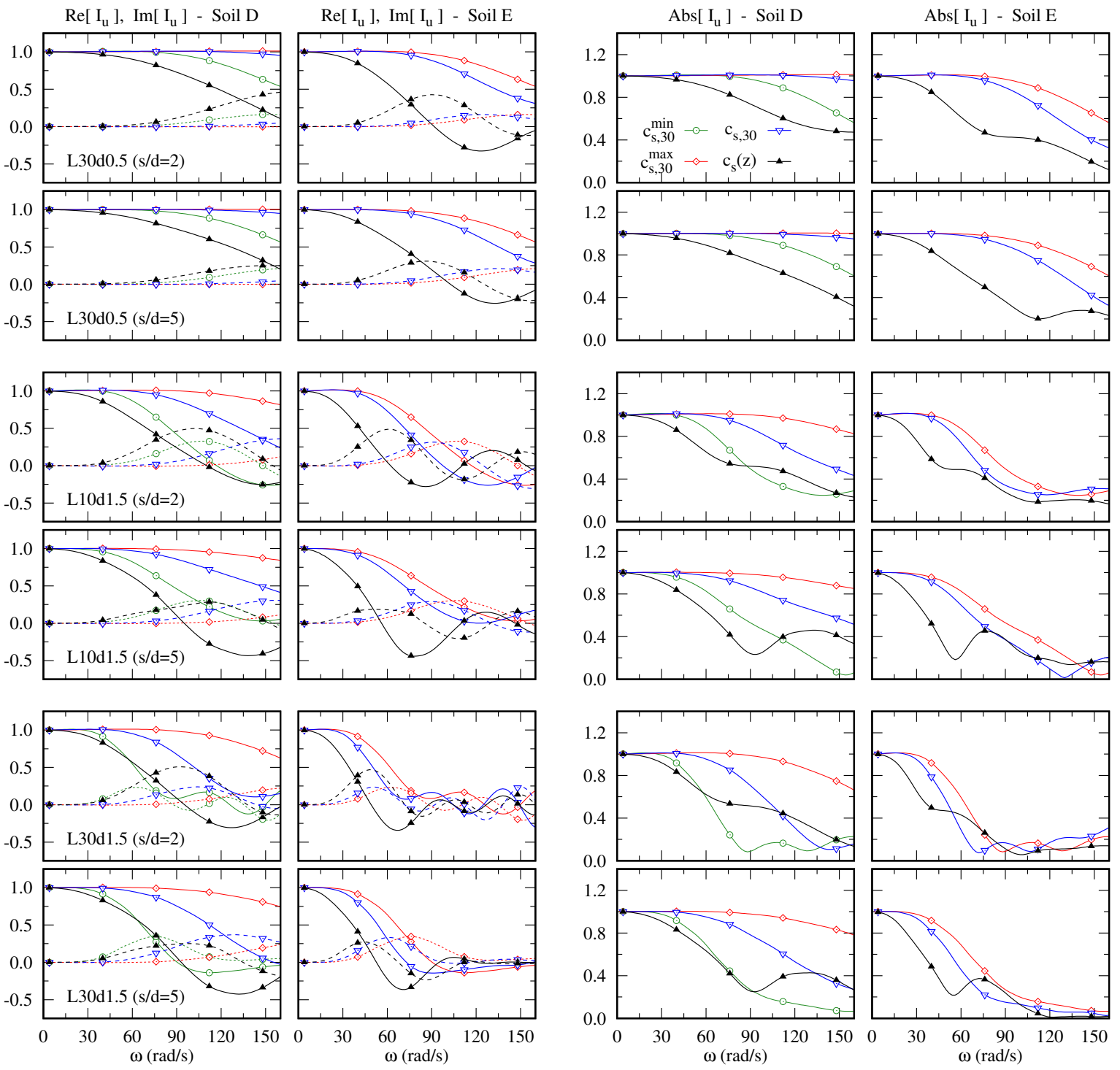

Figure 5: Translational kinematic interaction factors for $2 \times 2$ pile groups. (Dashed lines represent imaginary components) 

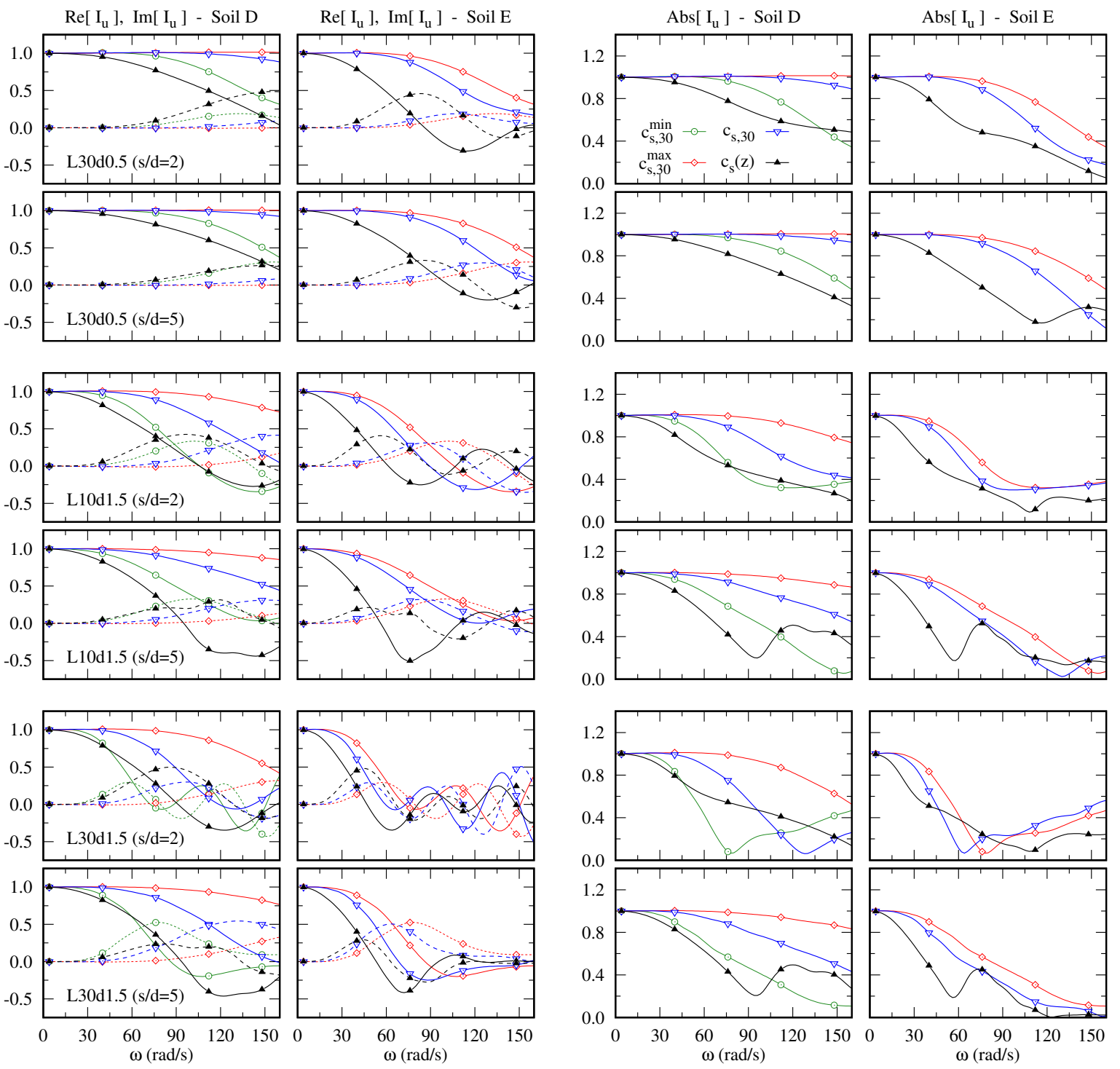

Figure 6: Translational kinematic interaction factors for $3 \times 3$ pile groups. (Dashed lines represent imaginary components) 

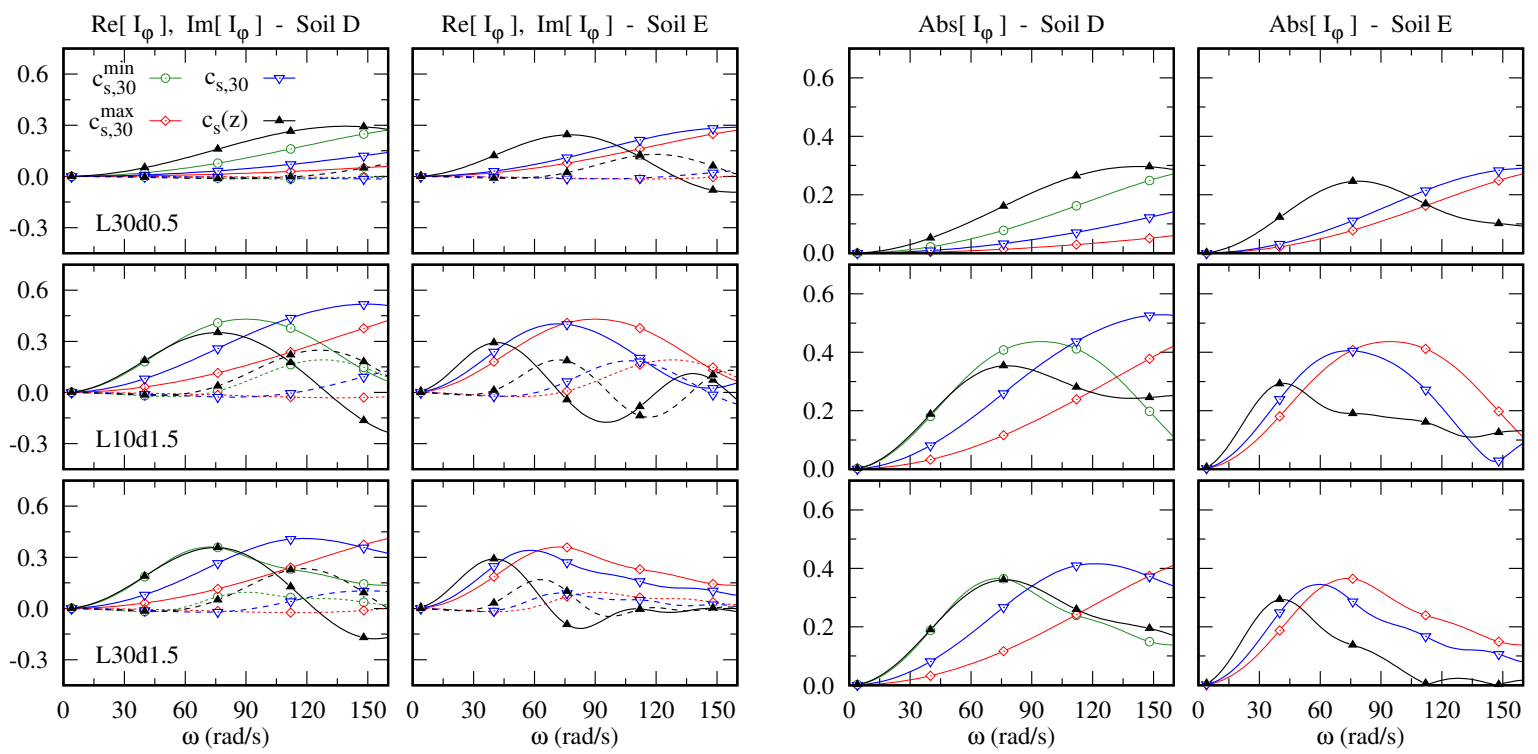

Figure 7: Rotational kinematic interaction factors for single piles. (Dashed lines represent imaginary components)

variable profiles present larger rotations at the pile head than their equivalent homogeneous soils. On the other hand, in the medium-high-frequency zone the real and imaginary components of the variable profiles decrease their values, changing their signs for large frequencies. This significant sign change is not produced for any of the homogeneous profiles in the studied frequency range. The two different zones can be also recognized in the absolute value of the rotational kinematic interaction factors. In the low-frequency range the $\left|I_{\varphi}\right|$ of the varying profiles exceed the ones of the $c_{s, 30}$ homogeneous profile; while, in the medium-high range, the non-homogeneous profiles present smaller rotations than the uniform soils. The frequencies that separate the two different zones depend on the diameter of the configuration and the soil type: approximately being $100 \mathrm{rad} / \mathrm{s}$ for $d=0.5 \mathrm{~m}$ and Soil E, and 90 or $42 \mathrm{rad} / \mathrm{s}$ for $d=1.5 \mathrm{~m}$ and Soil D or E, respectively.

For soil type E, and coinciding with what was found for the translational interaction factors, the $\left|I_{\varphi}\right|$ vanishes at frequencies over $110 \mathrm{rad} / \mathrm{s}$ for the $L 30 d 1.5$ configurations. Also, the singular behaviour of the $L 10 d 1.5$ configuration of presenting a higher response for the varying profile than for the equivalent homogeneous at high frequencies is also seen in the rotational factors.

The results for the $2 \times 2$ configurations are presented in Fig. 8, A significant reduction of the rotational kinematic interaction factors is found for the pile group, despite the value of $b$ that is used for the normalization augments with respect to the one of the single pile. This reduction is a well-known effect, which is produced by the vertical stiffness of the piles that restricts the rotation of the cap. Only the configuration $\operatorname{Li0d1.5}$ (shortest piles) presents results on the order of the ones of the single pile. Note that the same range is kept for all the figures that display the same variable in order to ease the comparison between them. Nevertheless, the results for the group configuration exhibit analogous behaviours as the ones of the single pile, also presenting the two frequency zones that were commented above. 

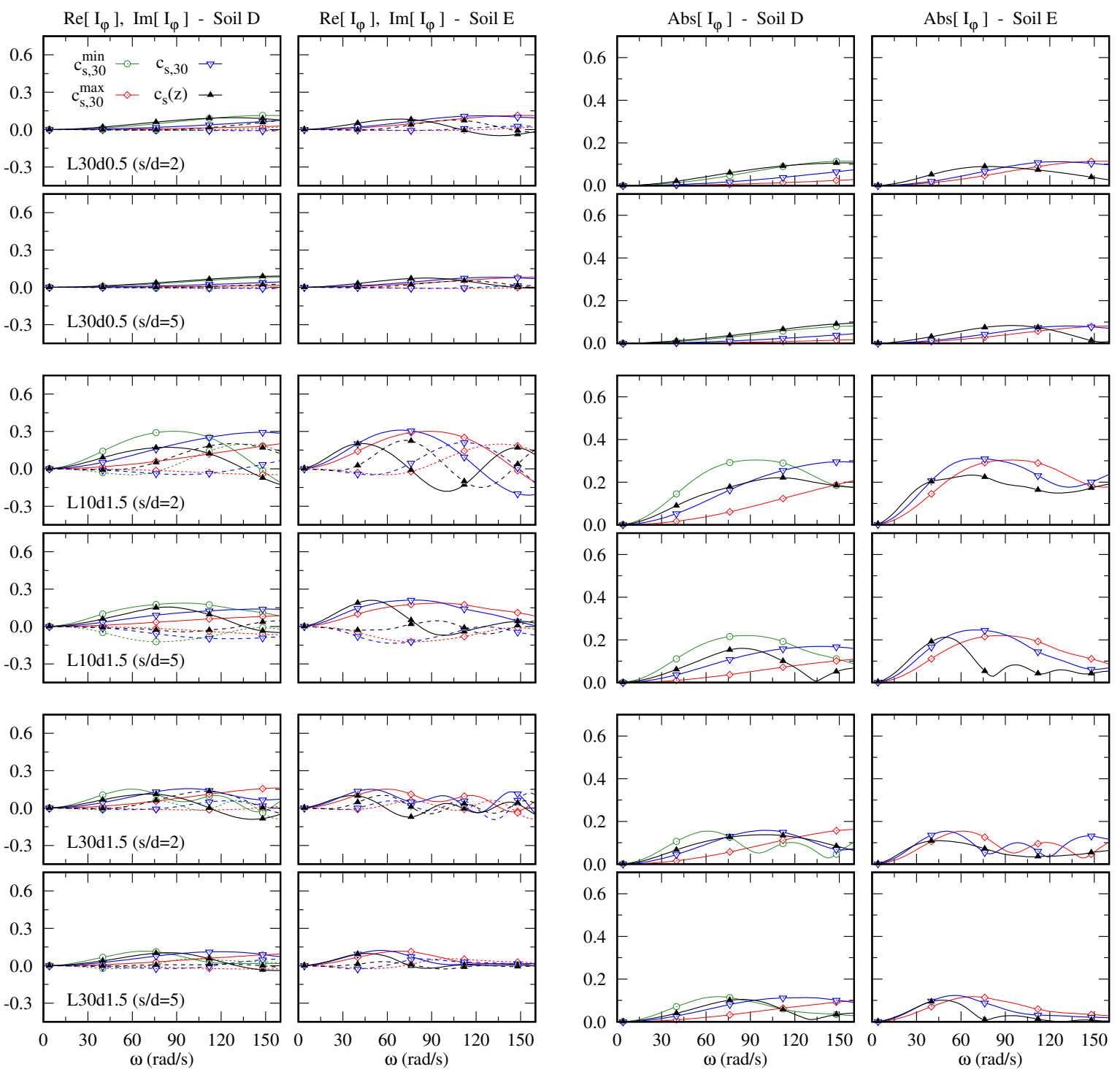

Figure 8: Rotational kinematic interaction factors for $2 \times 2$ pile groups. (Dashed lines represent imaginary components) 
Regarding the results corresponding to the foundations with closer piles $(s / d=2)$, the rotational interaction factors of the $L 30 d 1.5$ configuration again present an oscillatory behaviour in its real and imaginary components at large frequencies for soil type E. These oscillations are likewise found in the absolute values of the homogeneous soils, but not for the $\left|I_{\varphi}\right|$ of the variable profile. On the other hand, for soil type $\mathrm{D}$ this configuration $(L 30 d 1.5 \mathrm{~s} / \mathrm{d}=2)$ presents nearly the same rotational interaction factors for the nonhomogeneous and the equivalent homogeneous soils in terms of the absolute value, despite important differences can be observed between their real and imaginary components.

Attending to the effects of the distance between piles, increasing their separation slightly reduces the rotation of the group. The most noticeable difference between the results of the two $s / d$ values are found for the $d=1.5 \mathrm{~m}$ configurations: when the distance between the piles increases, the two frequency zones of different behaviours are appreciated in a clearer way, being the $\left|I_{\varphi}\right|$ values of the varying profiles lower than the ones of the equivalent homogeneous soil at large frequencies. In addition to this, and coinciding with what was found for the translational factors, the increment of the separation between the piles removes the high-frequency oscillations in the rotational interaction factors for the configurations L30d1.5 in soil E.

Finally, Fig. 9 displays the rotational kinematic interaction factors for the configurations of $3 \times 3$ pile groups. As found in the previous results, the increment in the number of piles drastically reduces the rotation of the cap. The shapes of the interaction factors of the $3 \times 3$ groups are similar to the ones of the $2 \times 2$ groups. The only aspect that is worthy of mention is the fact that for the L30d1.5 configuration the oscillations in the absolute value of $I_{\varphi}$ at large frequencies for the homogeneous profiles disappear and are replaced by an almost linear increase with frequency.

For these pile foundations, also increasing the separation between the piles further reduces the rotation at the center of the cap. However, the influence of the separation distance is lower than the one of the increment in the number of piles of the group.

\subsubsection{Kinematic interaction factors for soil type $\mathrm{C}$}

The previous sections have focused on the analysis of the kinematic interaction factors for relatively soft soils (type D and E), as those soils are normally the ones that require pile foundations in order to safely support constructions. However, the study of the effects of the soil profile variability has also been carried out for the soil type C. The results are not presented for brevity's sake, but the main conclusions of this analysis are briefly commented below.

Regarding the translational interaction factors, the results have practically a static behaviour $\left(I_{u} \approx 1\right)$ along the studied frequency range, so virtually no differences are found between the results of the variable and homogeneous profiles. On the other hand, the magnitude of the rotational kinematic interaction factors of the non-homogeneous soil significantly overtakes the one of the homogeneous profiles for all the frequency range. Those differences between the varying and constant profiles are produced only by the contribution of the real component of the interaction factors, as their imaginary component is almost negligible. Note that, as this soil type is stiffer than the ones studied in the previous section, only the behaviour of the low-frequency zone is seen for the considered frequency range. 

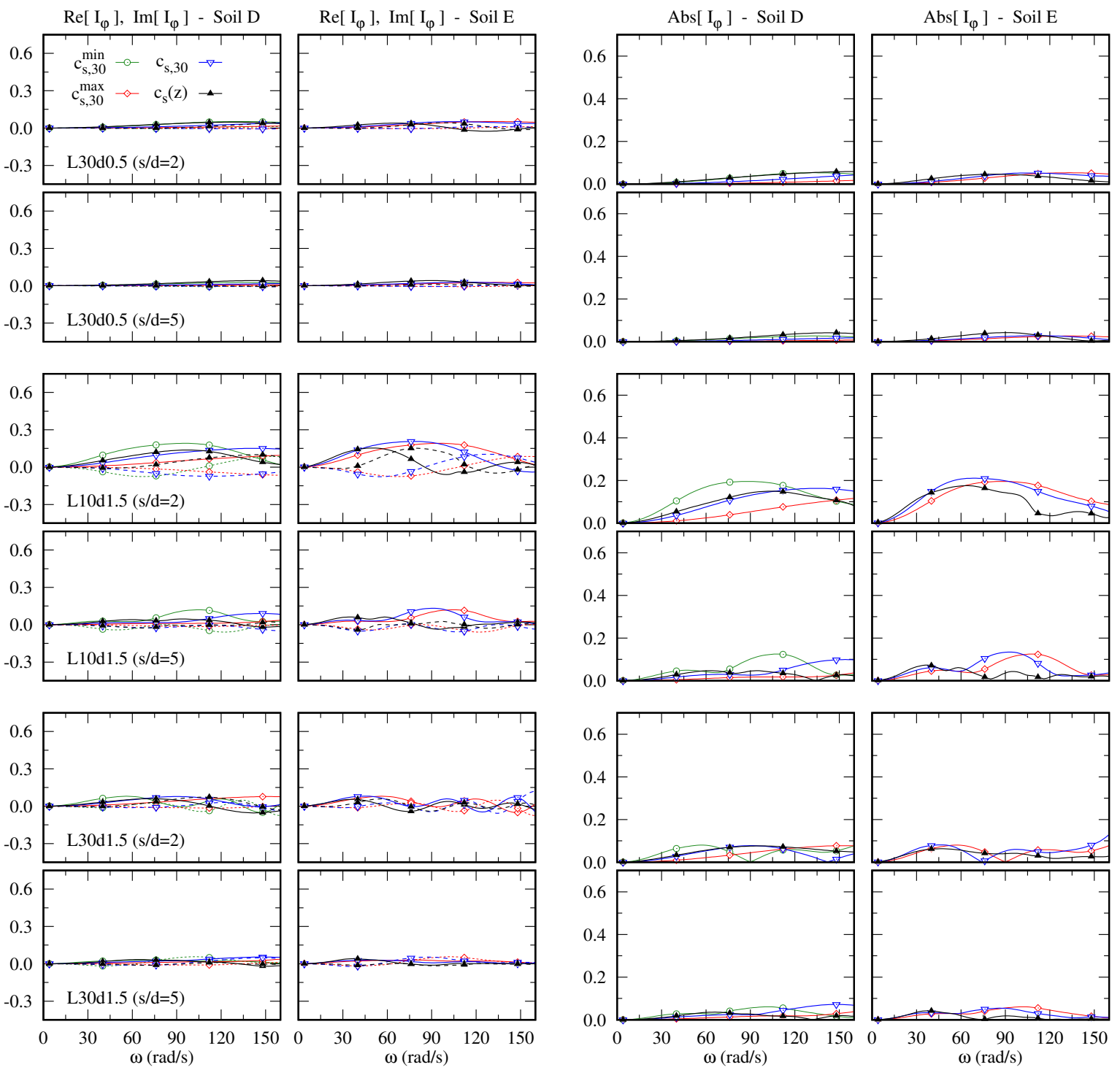

Figure 9: Rotational kinematic interaction factors for $3 \times 3$ pile groups. (Dashed lines represent imaginary components) 
Table 2: Real accelerograms used in the analyses.

\begin{tabular}{cccccc}
\hline \hline label & RSN $^{a}$ & Event Name & Year & Station Name & $c_{s, 30}(\mathrm{~m} / \mathrm{s})$ \\
\hline AD1 & 322 & Coalinga-01 & 1983 & Cantua Creek School & 275 (Type D) \\
AD2 & 766 & Loma Prieta & 1989 & Gilroy Array \#2 & 271 (Type D) \\
AD3 & 988 & Northridge-01 & 1994 & LA - Century City CC North & 278 (Type D) \\
AE1 & 178 & Imperial Valley-06 & 1979 & El Centro Array \#3 & 163 (Type E) \\
AE2 & 718 & Superstition Hills-01 & 1987 & Imperial Valley Wildlife Liquefaction Array & 179 (Type E) \\
AE3 & 729 & Superstition Hills-02 & 1987 & Imperial Valley Wildlife Liquefaction Array & 179 (Type E) \\
\hline \hline
\end{tabular}

Source: Data from the PEER NGA-West2 Database 24$]$.

${ }^{a}$ Record Sequence Number of the database.

Soil D
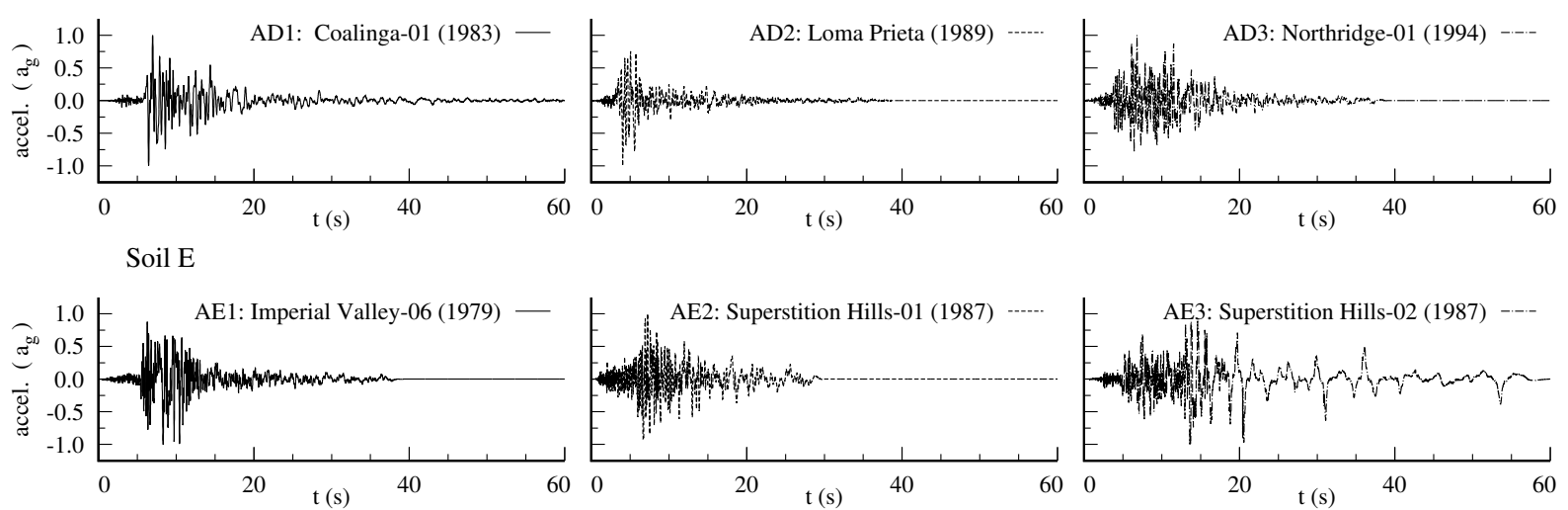

Figure 10: Time evolution of the real accelerograms used in the analyses.

\subsection{Pseudo-Spectral Accelerations}

In order to illustrate the effects that the differences in the kinematic interaction factors previously studied could have on the response of the supported structure, some results in terms of pseudo-spectral accelerations (PSA) are presented in this section. The structural time response is obtained from the kinematic interaction factors following the standard frequency-domain method [23]. For this purpose, three real accelerograms per soil type are used as seismic input. Those accelerograms are extracted from the PEER Ground Motion Database [24] and correspond to earthquake events produced in the California area with magnitudes between 6-7 and measured in stations located over soils of type $\mathrm{D}$ or E. The accelerations are scaled, so all signals present the same value of the ground maximum acceleration $a_{g}$. The structural response is computed in terms of this maximum acceleration. The information of the used accelerograms is presented in Table 2, while their time evolution is shown in Fig. 10,

These acceleration signals are assumed to be located at the free-surface level. In order to include the contribution of the rotational kinematic interaction factors in the spectra of maximum response, different structural height ratios $h / b$ are considered going from $h / b=0$ (neglecting the contribution of the cap rotation) to $h / b=10$. The results are presented not only in terms of the elastic response spectra for both the variable $\left(P S A_{c_{s}(z)}(T)\right)$ and equivalent homogeneous $\left(P S A_{c_{s, 30}}(T)\right)$ soil profiles, but also in terms 

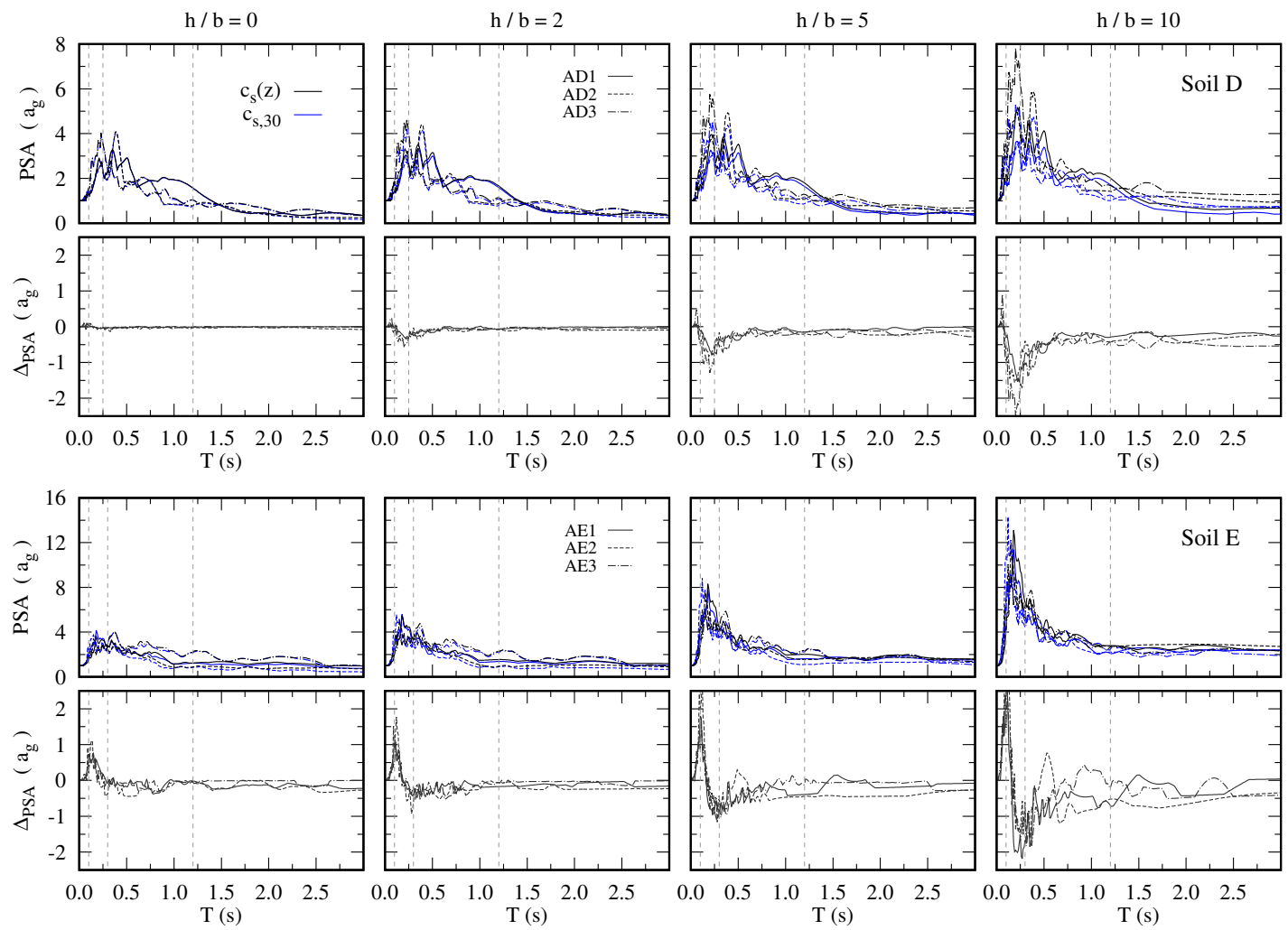

Figure 11: Elastic response spectra $(P S A)$ and differences between results of the equivalent homogeneous and non-homogeneous profiles $\left(\Delta_{P S A}\right)$. Single pile, configuration L30d1.5.

of the difference between them which is computed as:

$$
\Delta_{P S A}(T)=P S A_{c_{s, 30}}(T)-P S A_{c_{s}(z)}(T)
$$

where $T$ is the structural period in seconds. The difference $\Delta_{P S A}$ is defined in order to ease the analysis of the influence of the soil profile on the structural response. A positive value of $\Delta_{P S A}$ indicates that the homogeneous assumption is conservative; while a negative value implies that higher structural accelerations are obtained if the actual variable profile is considered. As mentioned before, both the response spectra and their differences are expressed in terms of the maximum ground acceleration of the excitation signal $a_{g}$.

Fig. 11 presents the elastic response spectra for the variable (black lines) and equivalent homogeneous (blue lines) profiles for a single pile configuration with $L 30 d 1.5$ embedded in soils $\mathrm{D}$ and $\mathrm{E}$, together with the corresponding differences $\Delta_{P S A}$. The results of the different accelerograms are indicated by different line styles according to Fig. 10.

The results can be explained with the aid of the absolute value of the kinematic interaction factors presented for this configuration in Figs. 4 and 7 , and noting that the major part of the energy of the earthquake excitation is found below $50 \mathrm{rad} / \mathrm{s}$. Attending to the $P S A$ obtained for soil type $\mathrm{D}$, the results of both profiles practically coincide if the contribution of the rotation of the cap is neglected $(h / b=0)$ as their translational interaction factors almost coincide for the $0-50 \mathrm{rad} / \mathrm{s}$ frequency range. However, as the 
height of the structure is increased, the differences between the non-homogeneous and the constant profile augment due to the contribution of the rotational interaction factor, which depends to a greater extent on the soil profile. These differences are specially relevant for periods around $0.25 \mathrm{~s}$, corresponding to systems with structural fundamental frequencies within the range of large energy content of the excitation. However, owing to the differences in the rotational kinematic interaction factors that are found even for $\omega \rightarrow 0$, the discrepancies between the PSA of the two profiles are extended to almost all the studied range for the extreme scenario of $h / b=10$. This effect of the $h / b$ parameter is also found for the soil type E. Moreover, even for the case of $h / b=0$ some differences between the results of the varying and equivalent homogeneous profiles can be seen. For this soil type and for low periods $(T \approx 0.1 \mathrm{~s})$ the accelerations of the homogeneous profile surpass the ones of the variable profile, what can be explained noting that the kinematic interaction factors are larger for the homogeneous profile at the frequency range corresponding to these periods $(\approx 60 \mathrm{rad} / \mathrm{s})$. Also, for soil E, the effects of the structural height on the magnitude of the $P S A$ are more important than for soil $\mathrm{D}$ (note the change of the ordinate-axis scale).

These effects of the soil profile on the structural maximum accelerations are also seen and in a clearer way attending to the results of $\Delta_{P S A}$. The homogeneous assumption tends to under-predict the structural maximum accelerations except for systems with very low periods. The highest differences between the two profiles are found around $T=0.25 \mathrm{~s}$, for which the equivalent homogeneous soil produces a maximum response that can be up to two times the peak ground acceleration lower than the maximum response of the variable profile.

Fig. 12 displays the differences between the pseudo-spectral accelerations $\Delta_{P S A}$ now for a group configuration of $3 \times 3$ piles with $L 30 d 1.5$. For this pile group, and because the $I_{\varphi}$ is drastically reduced when the number of piles increases, the effects of the structural height are almost negligible, being only appreciable for the largest height ratio $(h / b=10)$. The differences between the two profiles are more evident for the softer soil type (E), and for the groups with closer piles $(s / d=2)$. In general terms, for this configuration the equivalent homogeneous profiles produce higher acceleration values than the variable soils for low periods. However, negative values of $\Delta_{P S A}$ exist for soil type $\mathrm{E}$ along the whole period range which can be significant.

The results presented in Figs. 11 and 12 illustrate the general trends that are obtained for all studied configurations. However, more detailed information can be consulted in Tables 3 (soil type D) and 4 (soil type E). Those tables contain the minimum value of $\Delta_{P S A}$ that is obtained for all the representative configurations separated into different period intervals. The period division is done according to the one proposed in the Eurocode [22] for the definition of the Type 2 spectra for each ground type. These results allow to estimate the importance of considering the variability of the soil profile when studying the structural maximum acceleration response under different scenarios.

Attending to the results, and summarizing all the previous discussion, the soil type E presents higher differences between the non-homogeneous and equivalent homogeneous profiles. These differences are magnified as the structural height ratio augments due to the higher contribution of the rotational kinematic interaction factors to the structural response. Thus, including the $I_{\varphi}$ of the foundation is found to increase the accelerations 

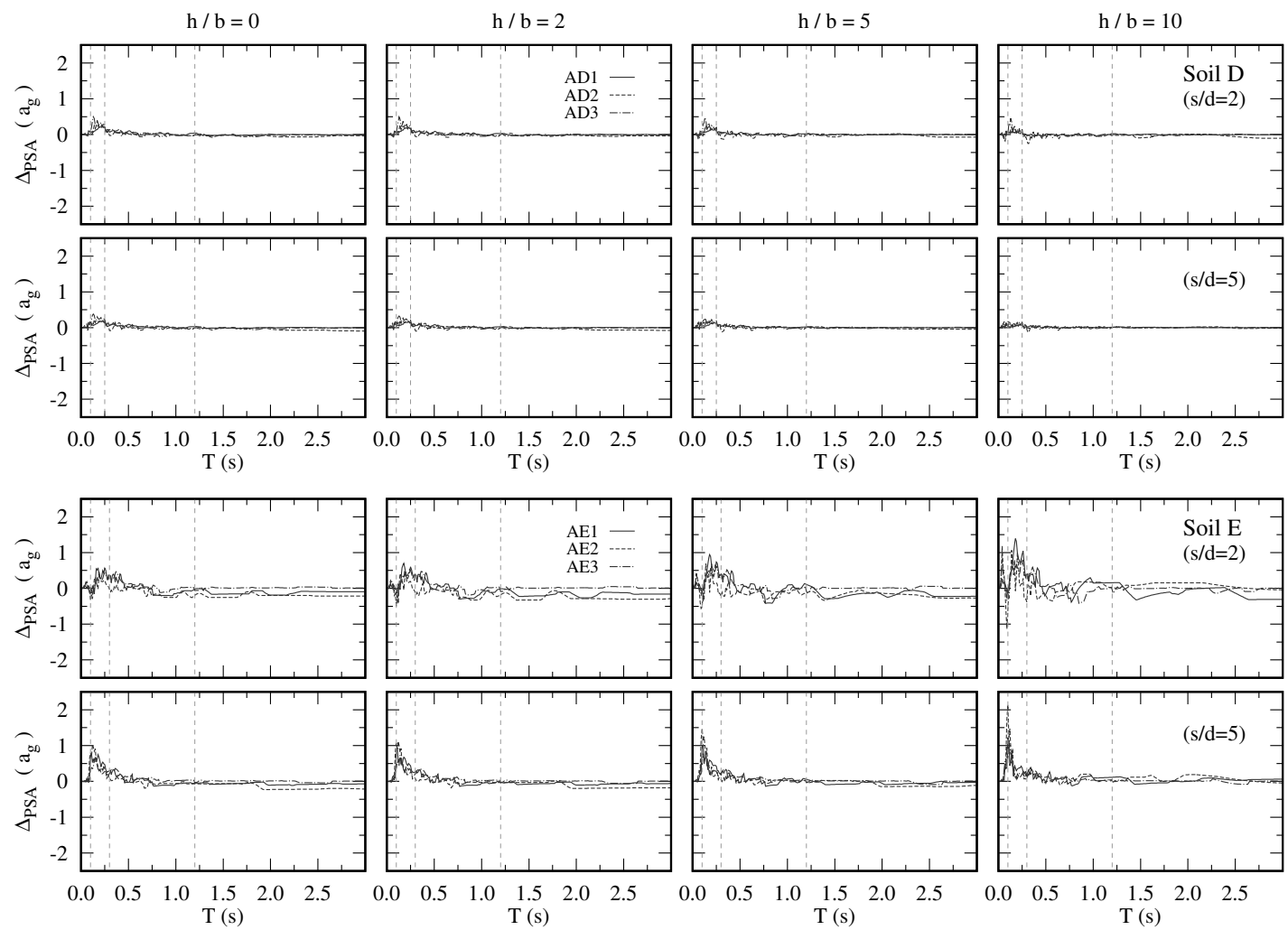

Figure 12: Differences between the elastic response spectra of the equivalent homogeneous and non-homogeneous profiles $\left(\Delta_{P S A}\right) .3 \times 3$ pile group, configuration L30d1.5. 
of the variable profile with respect to the constant one. This effect is explained attending to the rotational kinematic interaction factors presented above: in the low-frequency zone (which is the one that usually contains more energy of the seismic excitation) the variable profiles present higher rotations than their homogeneous equivalent ones. This effect is especially relevant for the single pile configuration, for which assuming the homogeneous profile can lead to a difference in the structural maximum accelerations with respect to the ones of the variable profile that can be over four times the peak ground acceleration. On the other hand, for pile groups these differences are generally not important (especially for soil type D), being the homogeneous assumption on the side of safety. However, for group configurations that present non-negligible cap rotations (i.e., closely spaced and small number of piles) founding slender structures, the variable profile can present maximum accelerations up to $1 a_{g}$ larger than the ones obtained by considering the equivalent homogeneous profile. Configurations with short piles (L10d1.5) are found to be the ones that present the most severe under-predictions of maximum accelerations among the pile groups under study.

\subsection{Differences in the Pile Response due to the Adoption of an Alternative Profile with Non-zero Shear Wave Velocity at the Soil Free-surface}

In the previous sections, the variable profile was defined following the power-law expressions presented in Table 1 based on the work of Wang and Wang [19]. As commented before, they proposed two types of regressions (power-law and linear) for the same experimental data. The choice of the firsts in this study was motivated by their better fitting to the empirical data. However, the possible drawback of the power-law expressions is that they present a zero shear wave velocity at the free-surface level. In this section, and with the intention of deepening the main thesis of the current work, some results obtained from the use of the linear expressions are presented. Fig. 13 shows the evolution with depth of the variable and homogeneous profiles for soil types D and E obtained through the use of the linear and power-law formulas. Note that the average shear wave velocities obtained for both types of expressions virtually coincide. This agreement was expected due to the elastodynamic nature of the fitting procedure used by Wang and Wang [19].

The absolute value of the lateral and rotational kinematic interaction factors for single piles embedded both in the linear variable profile and its equivalent homogeneous soil are presented in Fig. 14. In order to ease the comparison, the results of the power-law variable profile and its corresponding equivalent homogeneous profile already presented in Figs. 4 and 7 are also included. Note that the results of both equivalent homogeneous profiles are nearly the same as their shear wave velocities almost coincide. Regarding the results of the variable profiles, there are appreciable differences between the use of the linear or power-law expressions. The results for the linear profile are closer to the ones of the equivalent homogeneous soil due to the larger stiffness at the superficial layers if compared with the power-law medium (see Fig. 13). However, still a high influence of the variability of the soil profile is found both in the translational and rotational kinematic interaction factors.

On the other hand, Fig. 15 presents the results in terms of differences between the 
Table 3: Differences between the PSA of the equivalent homogeneous and non-homogeneous profiles $\Delta_{P S A}$ (units in $a_{g}$ ). Minimum values (unsafe homogeneous assuption) for different period ranges. Soil type D.

\begin{tabular}{|c|c|c|c|c|c|c|c|c|c|c|c|c|c|c|c|c|}
\hline \multirow[b]{2}{*}{ Configuration } & \multicolumn{4}{|c|}{$h / b=0$} & \multicolumn{4}{|c|}{$h / b=2$} & \multicolumn{4}{|c|}{$h / b=5$} & \multicolumn{4}{|c|}{$h / b=10$} \\
\hline & $T_{1}$ & $T_{2}$ & $T_{3}$ & $T_{4}$ & $T_{1}$ & $T_{2}$ & $T_{3}$ & $T_{4}$ & $T_{1}$ & $T_{2}$ & $T_{3}$ & $T_{4}$ & $T_{1}$ & $T_{2}$ & $T_{3}$ & $T_{4}$ \\
\hline \multicolumn{17}{|l|}{ L30d0.5 } \\
\hline single & -0.0 & -0.1 & -0.0 & -0.0 & $\underline{-0.2}$ & -0.2 & -0.1 & -0.0 & $\underline{-0.4}$ & -0.5 & -0.3 & -0.1 & -0.8 & -1.0 & -0.6 & -0.3 \\
\hline $2 \times 2, s / d=2$ & -0.0 & +0.0 & -0.0 & -0.0 & -0.0 & -0.0 & -0.0 & -0.0 & -0.1 & -0.1 & -0.1 & -0.0 & -0.1 & -0.3 & -0.2 & -0.1 \\
\hline $2 \times 2, s / d=5$ & -0.0 & +0.0 & -0.0 & -0.0 & -0.0 & +0.0 & -0.0 & -0.0 & -0.0 & -0.0 & -0.0 & -0.0 & -0.1 & -0.1 & -0.1 & -0.0 \\
\hline $3 \times 3, s / d=2$ & -0.0 & +0.0 & -0.0 & -0.0 & -0.0 & +0.0 & -0.0 & -0.0 & -0.0 & -0.0 & -0.0 & -0.0 & -0.1 & -0.1 & -0.1 & -0.0 \\
\hline $3 \times 3, s / d=5$ & -0.0 & +0.0 & -0.0 & -0.0 & -0.0 & +0.0 & -0.0 & -0.0 & -0.0 & +0.0 & -0.0 & -0.0 & -0.0 & -0.0 & -0.0 & -0.0 \\
\hline \multicolumn{17}{|l|}{ L10d1.5 } \\
\hline single & -0.1 & $\underline{-0.1}$ & -0.1 & -0.1 & -0.2 & -0.5 & $\underline{-0.3}$ & -0.1 & -0.3 & -1.3 & -0.7 & -0.3 & $\underline{-0.8}$ & -2.5 & -1.7 & -0.6 \\
\hline $2 \times 2, s / d=2$ & -0.1 & +0.1 & -0.1 & -0.1 & -0.1 & +0.0 & -0.1 & -0.1 & -0.1 & -0.2 & -0.2 & -0.1 & -0.1 & -0.6 & -0.5 & -0.2 \\
\hline $2 \mathrm{x} 2, s / d=5$ & $\underline{-0.1}$ & +0.1 & -0.1 & -0.1 & -0.1 & +0.0 & -0.1 & -0.1 & -0.0 & -0.0 & -0.1 & -0.0 & -0.1 & -0.3 & -0.2 & -0.1 \\
\hline $3 \times 3, s / d=2$ & -0.1 & +0.0 & -0.1 & -0.1 & -0.0 & +0.0 & -0.1 & -0.0 & -0.0 & -0.0 & -0.1 & -0.0 & -0.1 & -0.3 & -0.3 & -0.1 \\
\hline $3 \times 3, s / d=5$ & -0.1 & +0.1 & -0.1 & $\underline{-0.1}$ & -0.1 & +0.0 & -0.1 & -0.1 & -0.1 & +0.0 & -0.1 & -0.1 & -0.0 & -0.1 & -0.1 & -0.0 \\
\hline \multicolumn{17}{|l|}{ L30d1.5 } \\
\hline single & -0.1 & -0.1 & $\underline{-0.1}$ & -0.1 & -0.2 & $\underline{-0.6}$ & -0.3 & $\underline{-0.1}$ & -0.4 & $\underline{-1.3}$ & $\underline{-0.8}$ & $\underline{-0.3}$ & -0.8 & $\underline{-2.5}$ & $\underline{-1.7}$ & $\underline{-0.6}$ \\
\hline $2 \times 2, s / d=2$ & -0.1 & +0.0 & -0.1 & -0.1 & -0.1 & +0.0 & -0.1 & -0.1 & -0.1 & -0.0 & -0.2 & -0.1 & -0.1 & -0.3 & -0.4 & -0.1 \\
\hline $2 \times 2, s / d=5$ & -0.1 & +0.1 & -0.1 & -0.1 & -0.1 & +0.0 & -0.1 & -0.1 & -0.1 & +0.0 & -0.1 & -0.0 & -0.0 & -0.1 & -0.1 & -0.0 \\
\hline $3 \times 3, s / d=2$ & -0.0 & -0.0 & -0.1 & -0.1 & -0.1 & -0.0 & -0.1 & -0.1 & -0.1 & -0.0 & -0.1 & -0.1 & -0.2 & -0.0 & -0.2 & -0.1 \\
\hline $3 \times 3, s / d=5$ & -0.1 & +0.1 & -0.1 & -0.1 & -0.1 & +0.0 & -0.1 & -0.1 & -0.1 & +0.0 & -0.1 & -0.0 & -0.0 & +0.0 & -0.1 & -0.0 \\
\hline
\end{tabular}

Note: Bolded items correspond to the minimum value of the row. Underlined items correspond to the minimum value of the column.

Definition of the period ranges: $T_{1} \equiv T \in[0,0.1) \mathrm{s} ; T_{2} \equiv T \in[0.1,0.25) \mathrm{s} ; T_{3} \equiv T \in[0.25,1.2) \mathrm{s} ; T_{4} \equiv T \in[1.2,3] \mathrm{s}$. 
Table 4: Differences between the PSA of the equivalent homogeneous and non-homogeneous profiles $\Delta_{P S A}$ (units in $a_{g}$ ). Minimum values (unsafe homogeneous assuption) for different period ranges. Soil type E.

\begin{tabular}{|c|c|c|c|c|c|c|c|c|c|c|c|c|c|c|c|c|}
\hline \multirow[b]{2}{*}{ Configuration } & \multicolumn{4}{|c|}{$h / b=0$} & \multicolumn{4}{|c|}{$h / b=2$} & \multicolumn{4}{|c|}{$h / b=5$} & \multicolumn{4}{|c|}{$h / b=10$} \\
\hline & $T_{1}$ & $T_{2}$ & $T_{3}$ & $T_{4}$ & $T_{1}$ & $T_{2}$ & $T_{3}$ & $T_{4}$ & $T_{1}$ & $T_{2}$ & $T_{3}$ & $T_{4}$ & $T_{1}$ & $T_{2}$ & $T_{3}$ & $T_{4}$ \\
\hline \multicolumn{17}{|l|}{ L30d0.5 } \\
\hline single & -0.2 & -0.4 & -0.2 & -0.2 & $\underline{-0.9}$ & -0.9 & -0.4 & -0.3 & $\underline{-1.8}$ & $\underline{-2.2}$ & -0.8 & $\underline{-0.5}$ & $\underline{-3.3}$ & $\underline{-4.3}$ & -1.4 & $\underline{-0.8}$ \\
\hline $2 \times 2, s / d=2$ & -0.2 & -0.3 & -0.3 & -0.2 & -0.2 & -0.3 & -0.3 & -0.2 & -0.2 & -0.5 & -0.3 & -0.2 & -0.3 & -0.8 & -0.4 & -0.3 \\
\hline $2 \mathrm{x} 2, s / d=5$ & -0.1 & -0.2 & -0.3 & -0.2 & -0.1 & -0.2 & -0.2 & -0.2 & -0.1 & -0.3 & -0.3 & -0.2 & -0.2 & -0.4 & -0.2 & -0.2 \\
\hline $3 \times 3, s / d=2$ & -0.1 & -0.2 & -0.3 & -0.2 & -0.2 & -0.3 & -0.3 & -0.2 & -0.2 & -0.4 & -0.3 & -0.2 & -0.2 & -0.5 & -0.4 & -0.3 \\
\hline $3 \times 3, s / d=5$ & -0.0 & -0.2 & -0.2 & -0.2 & -0.0 & -0.2 & -0.2 & -0.1 & -0.1 & -0.2 & -0.2 & -0.1 & -0.0 & -0.2 & -0.2 & -0.1 \\
\hline \multicolumn{17}{|l|}{ L10d1.5 } \\
\hline single & -0.1 & -0.4 & $\underline{-0.6}$ & -0.3 & -0.1 & -0.9 & -0.6 & -0.2 & -0.1 & -1.1 & $\underline{-1.1}$ & -0.4 & -0.1 & -2.5 & -1.8 & -0.6 \\
\hline $2 \times 2, s / d=2$ & -0.0 & -0.1 & -0.3 & -0.3 & -0.1 & -0.5 & -0.5 & -0.3 & -0.0 & -0.8 & -0.5 & -0.1 & -0.0 & -0.9 & -0.9 & -0.2 \\
\hline $2 \times 2, s / d=5$ & -0.1 & -0.1 & -0.4 & -0.4 & -0.0 & -0.4 & -0.4 & -0.2 & -0.0 & -0.4 & -0.3 & -0.2 & -0.0 & -0.5 & -0.4 & -0.2 \\
\hline $3 \times 3, s / d=2$ & -0.1 & -0.1 & -0.3 & -0.3 & -0.0 & -0.4 & -0.5 & -0.3 & -0.0 & -0.5 & -0.5 & -0.1 & -0.0 & -0.5 & -0.6 & -0.1 \\
\hline $3 \times 3, s / d=5$ & -0.1 & -0.1 & -0.3 & $\underline{-0.4}$ & -0.0 & -0.3 & -0.4 & -0.3 & -0.0 & -0.3 & -0.4 & -0.3 & -0.0 & -0.5 & -0.4 & -0.2 \\
\hline \multicolumn{17}{|l|}{ L30d1.5 } \\
\hline single & -0.0 & $\underline{-0.4}$ & -0.4 & -0.3 & -0.0 & $\underline{-0.9}$ & $\underline{-0.6}$ & -0.3 & -0.0 & -1.2 & -1.0 & -0.5 & -0.0 & -2.2 & $\underline{-1.9}$ & -0.8 \\
\hline $2 \times 2, s / d=2$ & $\underline{-0.2}$ & -0.3 & -0.3 & -0.3 & -0.2 & -0.3 & -0.4 & $\underline{-0.5}$ & -0.2 & -0.4 & -0.5 & -0.3 & -0.4 & -0.7 & -0.6 & -0.3 \\
\hline $2 \times 2, s / d=5$ & -0.1 & +0.0 & -0.2 & -0.3 & -0.0 & -0.0 & -0.2 & -0.3 & -0.1 & -0.2 & -0.3 & -0.2 & -0.0 & -0.2 & -0.2 & -0.1 \\
\hline $3 \times 3, s / d=2$ & -0.1 & -0.3 & -0.3 & -0.3 & -0.2 & -0.5 & -0.3 & -0.3 & -0.6 & -0.5 & -0.4 & -0.3 & -1.1 & -0.6 & -0.4 & -0.3 \\
\hline $3 \times 3, s / d=5$ & -0.0 & +0.1 & -0.2 & -0.2 & -0.0 & +0.1 & -0.2 & -0.2 & -0.0 & +0.1 & -0.1 & -0.1 & -0.0 & +0.1 & -0.1 & -0.1 \\
\hline
\end{tabular}

Note: Bolded items correspond to the minimum value of the row. Underlined items correspond to the minimum value of the column.

Definition of the period ranges: $T_{1} \equiv T \in[0,0.1) \mathrm{s} ; T_{2} \equiv T \in[0.1,0.3) \mathrm{s} ; T_{3} \equiv T \in[0.3,1.2) \mathrm{s} ; T_{4} \equiv T \in[1.2,3] \mathrm{s}$. 
Soil D

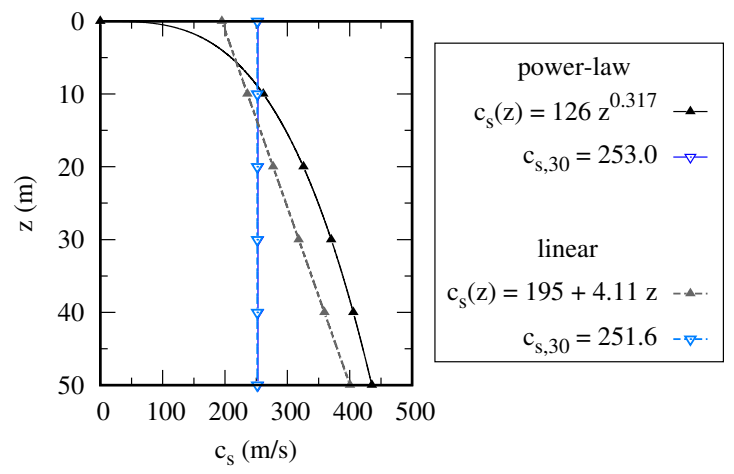

Soil E

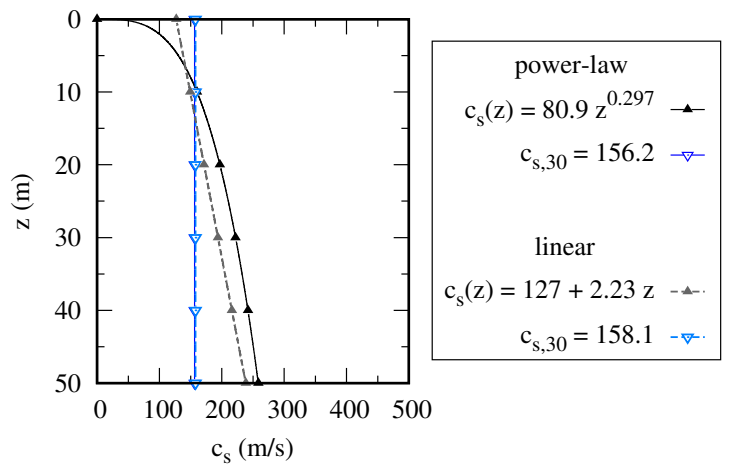

Figure 13: Evolution with depth of the power-law and linear variable profiles and their equivalent homogeneous soils. Expressions extracted from (Wang and Wang 2016).
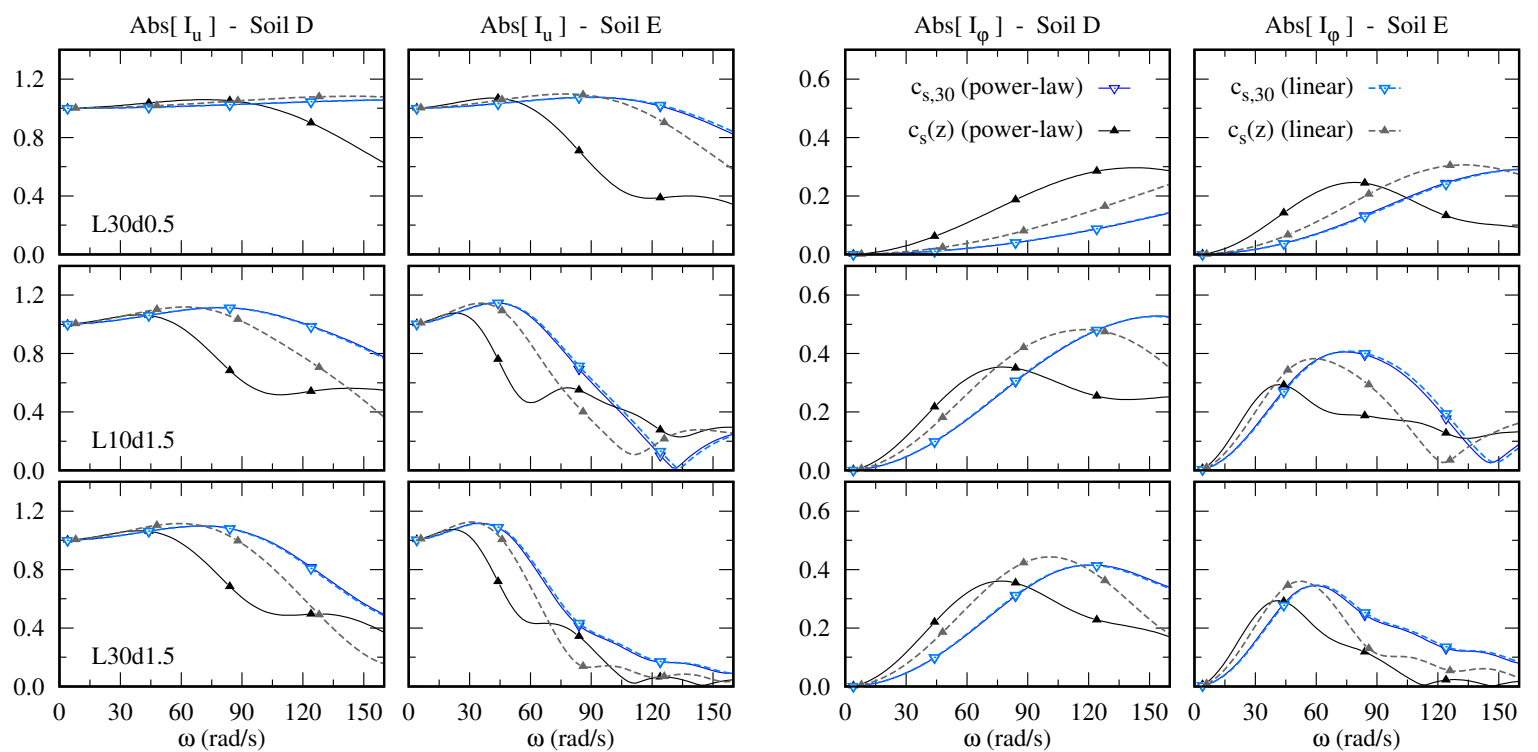

Figure 14: Translational and rotational kinematic interaction factors for single piles. Comparison between the use of the power-law or linear expressions for the variable profile. 

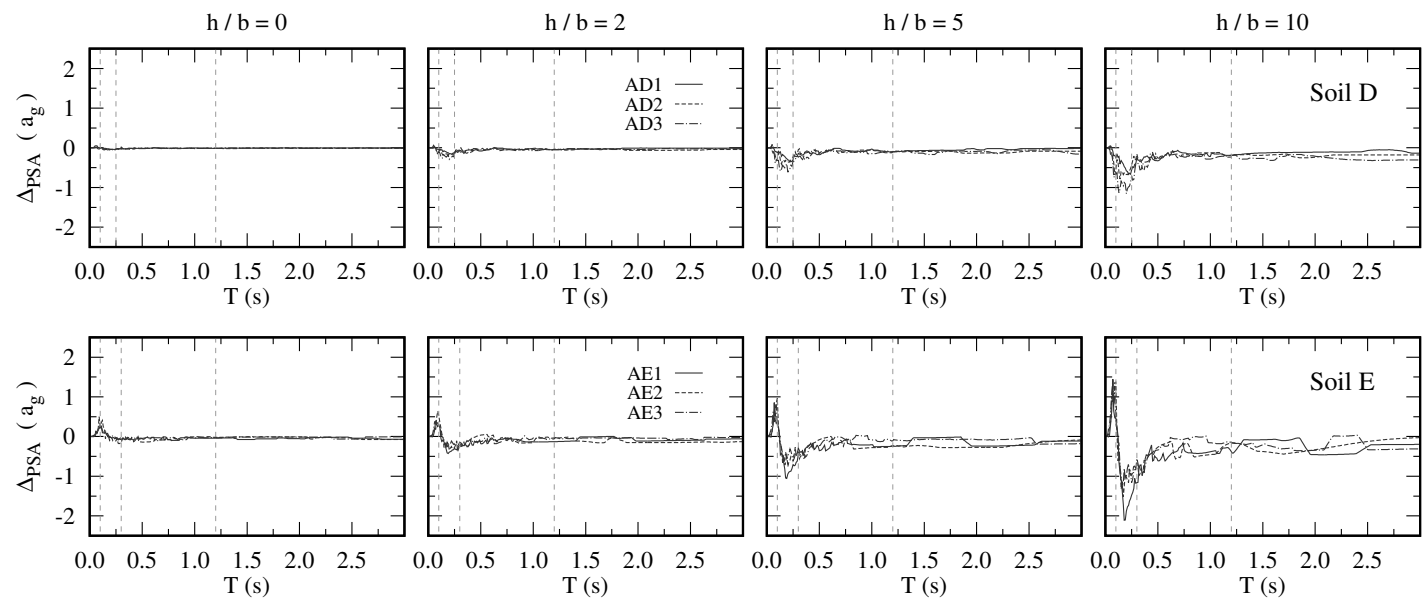

Figure 15: Differences between the elastic response spectra of the equivalent homogeneous and linear non-homogeneous profiles $\left(\Delta_{P S A}\right)$. Single pile, configuration L30d1.5.

pseudo-spectral accelerations of the linear variable profile and its equivalent homogeneous one. The selected pile dimensions are the same as the ones used in order to obtain the results displayed in Fig. 11, so that they can be compared. The influence of the linear variable profile with respect to the homogeneous media follows the same trend as the one obtained for the use of the power-law expressions.

Those results confirm the main conclusion of the current work. Despite presenting the same average shear wave velocity, profiles with different evolution-with-depth lead to different foundation responses. Thus, the equivalent homogeneous soil can not be used to reproduce the kinematic response of piles embedded in the variable-with-depth profiles.

Those results confirm the main conclusion of the current work. Despite presenting the same average shear wave velocity, profiles with different evolution-with-depth lead to different kinematic responses of the foundation. Therefore, in the cases studied, it is clear that the equivalent homogeneous soil can not be used as a replacement model to reproduce the real response. In this way, and depending on the problem to solve, a variable-with-depth model of the soil profile is necessary in order to obtain a result with an acceptable level of accuracy.

\section{Conclusions}

In this work, the influence of considering the variability of the soil profile on the kinematic interaction factors of several pile foundation is studied. For this purpose, representative variable-with-depth profiles corresponding to different types of soils are considered together with their equivalent homogeneous profile in terms of the average shear wave velocity $\left(c_{s, 30}\right)$. The time-harmonic seismic response of the pile foundation is obtained through an efficient numerical model based on the reciprocity theorem and the use of Green's functions for the layered half space. The effects of the soil profile on the structural maximum response are also analysed by computing the maximum response spectra from the studied kinematic interaction factors.

The main conclusions that are drawn from the obtained results are listed below: 
- Regarding the translational kinematic interaction factors $I_{u}$, the pile foundation filters to a great extent the soil seismic motion if the variable-with-depth profile is assumed. However, as the excitation frequency increases, there can be some ranges for which larger filtering effects are found for the equivalent homogeneous profile.

- On the contrary, the rotational kinematic interaction factors $I_{\varphi}$ increase in the low-frequency range if the variability of the soil profile is considered. In the highfrequency range, the rotations obtained for the equivalent homogeneous profile tend to be higher than those of the variable profile.

- These effects can be explained attending to the fact that the first layers of the variable profile are softer with respect to those of the equivalent homogeneous profile. Note that all the non-homogeneous profiles considered in this work have zero shear wave velocity at the free-surface level.

- Those differences between the two profiles in their kinematic interaction factors produce higher elastic response spectra for the homogeneous profile in the low period range $(T<0.15 \mathrm{~s})$. Thus, for that range, the homogeneous assumption is generally conservative in terms of the structural maximum accelerations.

- For larger structural periods, and especially in soft soils, the relevance of assuming the correct soil profile becomes more important. Appreciable differences between studied profiles are found for the whole period range, with the highest pseudospectral accelerations obtained when the variability of the soil profile is considered.

- The importance of including the soil variability further increases for those systems in which the foundation rotation has a high contribution to the structural response (i.e., slender structures and foundations with few closely spaced piles). The differences between the variable and homogeneous profiles can reach over $400 \%$ of the peak ground acceleration in the case of single piles or up to $100 \%$ for pile groups and the softest soil type.

\section{Acknowledgments}

The authors would like to thank Prof. Rafael Gallego for his constructive comments that undoubtedly improved the quality of the present work. This work was supported by the Subdirección General de Proyectos de Investigación of the Ministerio de Economía, Industria y Competitividad (MINECO) and Agencia Estatal de Investigación (AEI) of Spain and FEDER through research projects BIA2014-57640-R and BIA2017-88770-R. G.M. Álamo is recipient of FPU research fellowship FPU14/06115 from the Ministerio de Educación, Cultura y Deporte of Spain. The authors are grateful for this support.

\section{References}

[1] Fan, K., Gazetas, G., Kaynia, A., Kausel, E., Ahmad, S.. Kinematic seismic response of single piles and pile groups. J Geotech Eng 1991;117(12):1860-1879. doi:10.1061/(ASCE) 0733-9410(1991) 117:12(1860) 
[2] Makris, N., Gazetas, G.. Dynamic pile-soil-pile interaction. Part II: Lateral and seismic response. Earthq Eng Struct Dyn 1992;21(2):145-162. doi:10.1002/eqe.4290210204.

[3] Gazetas, G., Fan, K., Tazoh, T., Shimizu, K., Kavvadas, M., Makris, N.. Seismic pile-group-structure interaction. Geotechnical Special Publication 1992;:56-93.

[4] Anoyatis, G., Di Laora, R., Mandolini, A., Mylonakis, G.. Kinematic response of single piles for different boundary conditions: Analytical solutions and normalization schemes. Soil Dyn Earthq Eng 2013;44:183-195. doi:10.1016/j.soildyn.2012.09.011.

[5] Di Laora, R., Grossi, Y., de Sanctis, L., Viggiani, G.M.B.. An analytical solution for the rotational component of the foundation input motion induced by a pile group. Soil Dyn Earthq Eng 2017;97:424-438. doi:10.1016/j.soildyn.2017.03.027.

[6] Medina, C., Aznárez, J.J., Padrón, L.A., Maeso, O.. Effects of soil-structure interaction on the dynamic properties and seismic response of piled structures. Soil Dyn Earthq Eng 2013;53:160-175. doi:10.1016/j.soildyn.2013.07.004.

[7] Mamoon, S.M., Banerjee, P.K.. Response of piles and pile groups to travelling SHwaves. Earthq Eng Struct Dyn 1990;19(4):597-610. doi:10.1002/eqe.4290190410.

[8] Mamoon, S.M., Ahmad, S.. Seismic response of piles to obliquely incident SH, SV, and P waves. J Geotech Eng 1990;116(2):186-204. doi:10.1061/(ASCE) 0733-9410(1990) 116:2(186).

[9] Kaynia, A.M., Novak, M.. Response of pile foundations to rayleigh waves and obliquely incident body waves. Earthq Eng Struct Dyn 1992;21(4):303-318. doi:10.1002/eqe.4290210403.

[10] Makris, N., Badoni, D.. Seismic response of pile groups under obliqueshear and Rayleigh waves. Earthq Eng Struct Dyn 1995;24(4):517-532. doi:10.1002/eqe.4290240405.

[11] Giannakou, A., Gerolymos, N., Gazetas, G., Tazoh, T., Anastasopoulos, I.. Seismic behavior of batter piles: Elastic response. J Geotech Geoenviron Eng 2010;136(9):1187-1199. doi:10.1061/(ASCE) GT.1943-5606.0000337.

[12] Medina, C., Padrón, L.A., Aznárez, J.J., Santana, A., Maeso, O.. Kinematic interaction factors of deep foundations with inclined piles. Earthq Eng Struct Dyn 2014;43(13):2035-2050. doi:10.1002/eqe.2435.

[13] Rovithis, E., Mylonakis, G., Pitilakis, K.. Dynamic stiffness and kinematic response of single piles in inhomogeneous soil. Bull Earthq Eng 2013;11(6):1949-1972. doi:10.1007/s10518-013-9473-0.

[14] Kaynia, A.M., Kausel, E.. Dynamics of piles and pile groups in layered soil media. Soil Dyn Earthq Eng 1991;10(8):386-401. doi:10.1016/0267-7261(91)90053-3. 
[15] Álamo, G.M., Martínez-Castro, A.E., Padrón, L.A., Aznárez, J.J., Gallego, R., Maeso, O.. Efficient numerical model for the computation of impedance functions of inclined pile groups in layered soils. Eng Struct 2016;126:379-390.

[16] Pak, R.Y.S., Guzina, B.B.. Three-dimensional Green's functions for a multilayered half-space in displacement potentials. J Eng Mech 2002;128(4):449-461.

[17] Domínguez, J.. Boundary elements in dynamics. Southampton, NY: Computational Mechanics Publications \& Elsevier Applied Science; 1993.

[18] Padrón, L.A., Aznárez, J.J., Maeso, O.. Dynamic analysis of piled foundations in stratified soils by a BEM-FEM model. Soil Dyn Earthq Eng 2008;28(5):333-346. doi:10.1016/j.soildyn.2007.07.005.

[19] Wang, S., Wang, H.. Site-dependent shear-wave velocity equations versus depth in California and Japan. Soil Dyn Earthq Eng 2016;88:8-14. doi:10.1016/j.soildyn.2016.05.001.

[20] ASCE, . Minimum Design Loads for Buildings and Other Structures. ASCE/SEI 7-05. Reston, VA: American Society of Civil Engineers (ASCE); 2006.

[21] BSSC, . NEHRP recommended provisions for seismic regulations for new buildings and other structures (Report FEMA P-750). Washington, DC: Building Seismic Safety Council (BSSC); 2009.

[22] ECS, . Eurocode 8: Design of structures for earthquake resistance. Part 5: Foundations, Retaining Structures and Geotechnical Aspects. Brussels: European Committee for Standardization (ECS); 2004.

[23] Chopra, A.K.. Dynamic of structures. Theory and applications to earthquake engineering. NJ: Prentice-Hall; 2001.

[24] PEER, . NGA-West2 Ground Motion Database. 2013. <http://ngawest2.berkeley.edu/> (May 2017). 